\title{
Title
}

\section{The evolution of primate short-term memory}

\author{
Author Names and Affiliations \\ ManyPrimates (Group name as first author)
}

Géraud Aguenounon (Laboratoire de Neurosciences Cognitives et Adaptatives, UMR 7364, Université de Strasbourg, Strasbourg, France; Centre de Primatologie de l’Université de Strasbourg, Niederhausbergen, France)

Matthias Allritz (School of Psychology and Neuroscience, University of St Andrews, St Andrews, Scotland)

Drew M. Altschul (Department of Psychology, School of Philosophy, Psychology, and Language Sciences, The University of Edinburgh, Scotland

Sébastien Ballesta (Laboratoire de Neurosciences Cognitives et Adaptatives, UMR 7364, Université de Strasbourg, Strasbourg, France; Centre de Primatologie de l’Université de Strasbourg, Niederhausbergen, France)

Alice Beaud (Laboratoire de Neurosciences Cognitives et Adaptatives, UMR 7364, Université de Strasbourg, Strasbourg, France; Centre de Primatologie de l’Université de Strasbourg, Niederhausbergen, France)

Manuel Bohn* (Max Planck Institute for Evolutionary Anthropology, Leipzig, Germany) Sally L. Bornbusch (Department of Evolutionary Anthropology, Duke University, USA) 
Angela Brandão (Animal Behavior Department, Faculty of Science, University of Lisbon; Primate Cognition Research Group, Center for Philosophy of Sciences of the University of Lisbon, Portugal)

James Brooks (Wildlife Research Center, Kyoto University, Kyoto, Japan)

Thomas Bugnyar (Department of Behavioral \& Cognitive Biology, University of Vienna, Vienna, Austria)

Léa Bustamante (Laboratoire de Neurosciences Cognitives et Adaptatives, UMR 7364, Université de Strasbourg, Strasbourg, France; Centre de Primatologie de l'Université de Strasbourg, Niederhausbergen, France)

Josep Call (School of Psychology and Neuroscience, University of St Andrews, St Andrews, Scotland)

Charlotte Canteloup (Inkawu Vervet Project, South Africa; University of Lausanne, Lausanne, Switzerland) Chuangshi Cao (Wuhan Zoo, Wuhan, China)

Kai R. Caspar (Department of General Zoology, University of Duisburg-Essen, Essen, Germany) Diana da Silva (Primate Cognition Group, Lisbon) Alexandra A. de Sousa (Bath Spa University, UK)

Sarah E. DeTroy (Max Planck Institute for Evolutionary Anthropology, Leipzig, Germany) Shona Duguid (School of Psychology and Neuroscience, University of St Andrews, St Andrews, Scotland)

Timothy M. Eppley (Conservation Science \& Wildlife Health, San Diego Zoo Wildlife Alliance, San Diego, USA; Department of Anthropology, Portland State University, Portland, USA) 
Claudia Fichtel (Behavioral Ecology \& Sociobiology Unit, German Primate Center - Leibniz Institute for Primate Research, Göttingen, Germany)

Julia Fischer (Cognitive Ethology Laboratory, German Primate Center - Leibniz Institute for

Primate Research, Göttingen, Germany)

Chi Gong (Wuhan Zoo, Wuhan, China)

James A. Grange (School of Psychology, Keele University, UK)

Nicholas M. Grebe (Department of Evolutionary Anthropology, Duke University, USA)

Daniel Hanus (Max Planck Institute for Evolutionary Anthropology, Leipzig, Germany)

Daniel Haun (Max Planck Institute for Evolutionary Anthropology, Leipzig, Germany)

Lou M. Haux (Center for Adaptive Rationality, Max Planck Institute for Human Development,

Berlin, Germany)

Yseult Héjja-Brichard (Centre d'Ecologie Fonctionnelle et Evolutive, Univ Montpellier, CNRS, EPHE, IRD, Montpellier, France)

Annabella Helman (Department of Evolutionary Anthropology, Duke University, USA)

Istvan Hernadi (UP-Gedeon Richter Grastyan Translational Research Center, University of Pecs, Pecs, Hungary)

R. Adriana Hernandez-Aguilar (Department of Social Psychology and Quantitative Psychology, University of Barcelona, Serra Húnter Program, Barcelona, Spain \& Centre for Ecological and Evolutionary Synthesis, University of Oslo, Norway)

Esther Herrmann (Centre for Comparative and Evolutionary Psychology, Department of Psychology, University of Portsmouth, United Kingdom) 
Lydia M. Hopper (Lester E. Fisher Center for the Study and Conservation of Apes, Lincoln Park Zoo, Chicago, USA \& Department of Molecular and Comparative Pathobiology, Johns Hopkins University School of Medicine, Baltimore, USA)

Lauren H. Howard (Department of Psychology, Scientific and Philosophical Studies of the Mind Program, Biological Foundations of Behavior Program, Franklin \& Marshall College, Lancaster,

\author{
PA, USA) \\ Lei Huang (Wuhan Zoo, Wuhan, China)
}

Sarah M. Huskisson (Lincoln Park Zoo, Chicago, USA)

Ivo Jacobs (Department of Philosophy / Cognitive Science, Lund University, Sweden; Lund

University Primate Research Station Furuvik, Sweden)

Zhiyong Jin (Shanghai Key Laboratory of Brain Functional Genomics, Key Laboratory of Brain Functional Genomics Ministry of Education, Affiliated Mental Health Center (ECNU), School of Psychology and Cognitive Science, East China Normal University, Shanghai, 200062, China)

Marine Joly (Centre for Comparative and Evolutionary Psychology, Department of Psychology, University of Portsmouth, UK)

Fumihiro Kano (University of Konstanz, Konstanz, Germany; Kumamoto Sanctuary, Kumamoto, Japan)

Stefanie Keupp (German Primate Center and Leibniz ScienceCampus Primate Cognition, Göttingen, Germany)

Evelin Kiefer (UP-Gedeon Richter Grastyan Translational Research Center, University of Pecs, Pecs, Hungary)

Balázs Knakker (UP-Gedeon Richter Grastyan Translational Research Center, University of Pecs, Pecs, Hungary) 
Katalin Kóczán (UP-Gedeon Richter Grastyan Translational Research Center, University of Pecs, Pecs, Hungary)

Larissa Kraus (Max Planck Institute for Evolutionary Anthropology, Leipzig, Germany) Sze Chai Kwok (Shanghai Key Laboratory of Brain Functional Genomics, Key Laboratory of Brain Functional Genomics Ministry of Education, Shanghai Key Laboratory of Magnetic Resonance, Affiliated Mental Health Center (ECNU), School of Psychology and Cognitive Science, East China Normal University, Shanghai, China; Division of Natural and Applied Sciences, Duke Kunshan University, Kunshan, Jiangsu, China; Shanghai Changning Mental Health Center, China)

Marie Lefrançois (Laboratoire de Neurosciences Cognitives et Adaptatives, UMR 7364, Université de Strasbourg, Strasbourg, France; Centre de Primatologie de l’Université de Strasbourg, Niederhausbergen, France)

Laura Lewis (Department of Human Evolutionary Biology, Harvard University, USA) Siyi Liu (School of Psychological and Cognitive Sciences, Beijing Key Laboratory of Behavior and Mental Health, Peking University, Beijing 100871, China)

Miquel Llorente (Serra Húnter Fellow, Department of Psychology, University of Girona, Girona, Spain)

Elizabeth Lonsdorf (Department of Psychology, Biological Foundations of Behavior Program, Franklin \& Marshall College, Lancaster, PA, USA)

Louise Loyant (Centre for Comparative and Evolutionary Psychology, Department of Psychology, University of Portsmouth, UK)

Katarzyna Majecka (The Natural History Museum, Faculty of Biology and Environmental Protection, University of Lodz, Poland) 
Luke Maurits (Max Planck Institute for Evolutionary Anthropology, Leipzig, Germany)

Hélène Meunier (Laboratoire de Neurosciences Cognitives et Adaptatives, UMR 7364, Université de Strasbourg, Strasbourg, France; Centre de Primatologie de l'Université de Strasbourg, Niederhausbergen, France)

Flávia Mobili (Evolutionary Cognition Group, University of Zurich, Switzerland)

Luca Morino (Muséum National d'Histoire Naturelle, Paris, France)

Alba Motes-Rodrigo (Department of Ecology and Evolution, University of Lausanne, Switzerland)

Vincent Nijman (School of Social Science, Oxford Brookes University, Oxford, UK)

Caroline Nkoy Ihomi (Laboratoire de Neurosciences Cognitives et Adaptatives, UMR 7364,

Université de Strasbourg, Strasbourg, France; Centre de Primatologie de l'Université de Strasbourg, Niederhausbergen, France)

Tomas Persson (Department of Philosophy / Cognitive Science, Lund University, Sweden; Lund University Primate Research Station Furuvik)

Dariusz Pietraszewski (Department of Ecology and Vertebrate Zoology, Faculty of Biology and Environmental Protection, University of Lodz, Poland)

Juan Felipe Reátiga Parrish (Laboratoire de Neurosciences Cognitives et Adaptatives, UMR 7364, Université de Strasbourg, Strasbourg, France; Centre de Primatologie de l’Université de Strasbourg, Niederhausbergen, France)

Anthony Roig (Centre de Primatologie de l'Université de Strasbourg, Niederhausbergen, France; Normandie University, UNIROUEN, CRFDP (EA7475), Rouen, France) Alejandro Sánchez-Amaro (Department of Comparative Cultural Psychology, Max Planck Institute for Evolutionary Anthropology, Leipzig, Germany) 
Yutaro Sato (Wildlife Research Center, Kyoto University, Kyoto, Japan)

Gabriela-Alina Sauciuc (Department of Philosophy / Cognitive Science, Lund University,

Sweden; Lund University Primate Research Station Furuvik)

Allie E. Schrock (Department of Evolutionary Anthropology, Duke University, USA)

Manon K. Schweinfurth (School of Psychology and Neuroscience, University of St Andrews, St

Andrews, Scotland)

Amanda Seed (School of Psychology and Neuroscience, University of St Andrews, St Andrews, Scotland)

Caroline L. Shearer (Department of Evolutionary Anthropology, Duke University, USA) Vedrana Šlipogor (Department of Zoology, Faculty of Science, University of South Bohemia, Budweis, Czech Republic; Department of Behavioral and Cognitive Biology, Faculty of Life Sciences, University of Vienna, Vienna, Austria)

Yanjie Su (School of Psychological and Cognitive Sciences, Beijing Key Laboratory of Behavior and Mental Health, Peking University, Beijing 100871, China)

Kirsten Sutherland (Max Planck Institute for Evolutionary Anthropology, Leipzig, Germany) Jingzhi Tan (Institute of Psychology, Leiden University, the Netherlands)

Derry Taylor (Institute of Biology, University of Neuchâtel, Switzerland)

Camille A. Troisi (Department of Experimental Psychology, Ghent University, Belgium)

Christoph J. Völter (Comparative Cognition, Messerli Research Institute, University of Veterinary Medicine Vienna, Medical University of Vienna and University of Vienna, Vienna, Austria; School of Psychology and Neuroscience, University of St Andrews, St Andrews, Scotland)

Elizabeth Warren (University of St. Andrews, St Andrews, Scotland) 


\section{Julia Watzek}

Pauline Zablocki-Thomas (California National Primate Research Center, Davis, CA, USA)

* Correspondence should be addressed to Manuel Bohn (manyprimates@gmail.com) 


\begin{abstract}
Short-term memory is implicated in a range of cognitive abilities and is critical for understanding primate cognitive evolution. To investigate the effects of phylogeny, ecology and sociality on short-term memory, we tested the largest and most diverse primate sample to date (421 non-human primates across 41 species) in an experimental delayed-response task. Our results confirm previous findings that longer delays decrease memory performance across species and taxa. Our analyses demonstrate a considerable contribution of phylogeny over ecological and social factors on the distribution of short-term memory performance in primates; closely related species had more similar short-term memory abilities. Overall, individuals in the branch of Hominoidea performed better compared to Cercopithecoidea, who in turn performed above Platyrrhini and Strepsirrhini. Interdependencies between phylogeny and socioecology of a given species presented an obstacle to disentangling the effects of each of these factors on the evolution of short-term memory capacity. However, this study offers an important step forward in understanding the interspecies and individual variation in short-term memory ability by providing the first phylogenetic reconstruction of this trait's evolutionary history. The dataset constitutes a unique resource for studying the evolution of primate cognition and the role of short-term memory in other cognitive abilities.
\end{abstract}

Keywords: cognitive evolution, short-term memory, primate cognition, phylogenetic analysis 


\section{Introduction}

Inferring how, why, and when psychological traits evolved is a goal central to the field of comparative cognition (MacLean et al., 2012). The cognitive capacities of non-human primates ("primates" hereafter) have particular relevance for understanding the emergence of human cognition (MacLean, 2016). A systematic, comparative analysis of behaviors in extant primates is the best tool available to infer cognitive characters in ancestral taxa, and accordingly estimate when and in which lineages particular cognitive traits emerged and how they changed over the course of evolution (Shettleworth, 2010). Compared to many other mammals, primates have evolved enlarged brains relative to body size (Boddy et al., 2012) and higher neuronal densities (HerculanoHouzel et al., 2007). Consistent with this remarkable neural architecture, many primate species have been found to display advanced skills in various domains such as memory (Ghazizadeh et al., 2018; Lewis et al., 2019), tool making and use (Resende et al., 2021; Sanz et al., 2013; Shumaker et al., 2011; Wynn et al., 2011), planning (Osvath, 2009; Prétôt \& Brosnan, 2019), causal reasoning (Cacchione \& Rakoczy, 2017; Völter \& Call, 2017), theory of mind (Crockford et al., 2012; Krupenye \& Call, 2019), and metacognition (Basile et al., 2015; Beran et al., 2015; Rosati \& Santos, 2016).

What social and ecological forces drive the evolution of these complex cognitive traits in primates? Popular hypotheses have highlighted the particular demands associated with facing ecological variation and living in a dynamic social environment. The Ecological Intelligence Hypothesis (Milton, 1981; Rosati, 2017) posits that living in a structurally complex habitat partly drives the evolution of specialized and advanced cognitive skills in primates, for reasons including: 1) memorizing numerous locations and anticipating when key food resources are available is an 
important fitness advantage for primates (Milton, 1981);2) living in an unpredictable environment requires a certain degree of cognitive flexibility to regularly discover new resources or develop new behavioral strategies in order to cover minimal maintenance costs (Sol, 2009); and 3) developing technical skills (e.g. extractive foraging, tool use) offers the opportunity to get access to exclusive and/or calorie-rich resources (e.g. nuts or social insects) (Byrne, 1997).

The Social Intelligence Hypothesis, and derived variations thereof, argues that group-living and the subsequent need to compete and/or cooperate with conspecifics are key drivers shaping the evolution of primate cognition (Burkart et al., 2009; Byrne \& Whiten, 1988; Dunbar, 1998; Dunbar \& Shultz, 2007; Jolly, 1966; Tomasello \& Call, 1997). Most primates are social and live in groups ranging from two to hundreds of individuals (Smuts et al., 1986). Being able to track individual relationships and recognise allies and competitors is thus crucial to foraging, mating, and rearing offspring. Note that the two hypotheses are not mutually exclusive, specifically in the context of the competition over food.

Hypotheses regarding the drivers of cognitive and brain evolution can be fruitfully tested by comparing different primate species with respect to their cognitive abilities. To succeed, this approach requires datasets that span a large number of species living in different ecological and social environments. Primates are well-suited to such an inquiry by virtue of their diverse diets (ranging from frugivory, to folivory and omnivory; DeCasien, et al., 2017) and social systems (ranging from group living, to pair living, and more solitary lifestyles; Kappeler, 1999). However, such datasets are not readily available due to the logistic difficulties associated with testing large numbers of primates and, accordingly, little empirical research has explicitly tested these 
hypotheses (Hopper et al., 2018). Indeed, a recent review found that only a fraction of comparative primate cognition studies include more than two species (ManyPrimates, 2019a). Collaborative, multi-site research efforts with multiple species are thus required to address these types of evolutionary and phylogenetic questions. In this study, we leveraged a previously established infrastructure (ManyPrimates, 2019b, see also below) and tested one of the largest and most diverse datasets ever collected using a standardized experimental procedure. We investigated the evolution of one of the most fundamental cognitive abilities in primates: short-term memory.

The concept of short-term memory (STM) - the ability to hold active representations over short periods of time - has played a central role in psychological research for centuries and can be traced to the very roots of modern psychology (Atkinson \& Shiffrin, 1971). STM is usually contrasted with working memory, which captures the ability to mentally manipulate such representations (Cowan, 2008). Neuroscientific studies in humans suggest that STM is also distinct from other memory capacities such as long-term memory (Vallar \& Papagno, 2002). It has been argued that STM is implicated in almost all cognitive tasks (Jonides et al., 2008). For instance, success on object permanence tasks requires remembering the location of hidden rewards after short delays (Zewald \& Jacobs, 2021). From an evolutionary perspective, researchers have suggested that constraints on STM could explain species differences in cognitive abilities, such as a lack of recursive communication and complex tool-use in many primate species (Read, 2008). As such, understanding the origins of STM and its phylogenetic distribution represents an important goal for comparative psychology. 
One key source of STM failure (i.e. forgetting) stems from time-based decay of mental representations. Thus, one valuable methodological tool for evaluating STM capacity is by varying retention intervals in a task (Barth \& Call, 2006; Harlow et al., 1932; Maslow \& Harlow, 1932; Nissen et al., 1938). For example, Mercer \& Mckeown (2014) presented (human) participants with two tones that they had to compare and implemented several conditions varying the interval of time between the presentation of the tones. The authors observed a significant drop in performance as the interval length increased. In the context of comparative cognition studies, retention interval often refers to the length of time between seeing an object hidden in a specific location and the subsequent testing of memory for the object's location. Empirical studies on STM in humans show that, during the retention interval of STM tasks, areas of the prefrontal cortex show elevated activity levels, suggesting that STM representations are supported by neural activity in these areas (Grimault et al., 2009; Postle, 2015; Riley \& Constantinidis, 2016).

Since the initial study by Hunter (1913) on "delayed reactions," the study of STM in primates has largely focused on only a few species, especially rhesus macaques (Macaca mulatta) (but see Harlow et al., 1932; Harlow \& Bromer, 1939). STM in rhesus macaques is underpinned by neural mechanisms similar to those observed in humans (Constantinidis \& Procyk, 2004) and is distinct from other memory capacities such as long-term memory (Zola-Morgan \& Squire, 1986). While rhesus macaques' performance on STM tasks is significantly lower than human performance, there is evidence that patterns of primate STM parallel those in humans; for example, items presented early or late in a test set are better recalled than those in the middle (the "serial position" effect; Sands \& Wright, 1980). Additionally, research with chimpanzees (Pan troglodytes), suggests that their STM is constrained in ways comparable to the 'magic number seven' (plus or minus 2) effect 
in humans, wherein humans often struggle in STM tasks that require subjects to recall more than seven items (Miller, 1956). In research using touch-screen technology to order numbers that became hidden after a short delay, chimpanzees' STM capacity was found to be between 4 and 9 items (Inoue \& Matsuzawa, 2009; Kawai \& Matsuzawa, 2000).

It is clear that primate species vary in their STM performance. However, due to the wide variety of methods used to study this phenomenon, it is difficult to systematically assess cross-species similarities and differences in STM from the published literature. Additionally, due to the fact that only $15 \%$ of the approximately 500 primate species have been included in studies of primate cognition, and only 19\% of recent studies have included more than one species (ManyPrimates et al., 2019a), previous work has not been able to answer questions about evolutionary processes that account for species differences in STM. To address these limitations, in this study, we aimed to use a single test method to evaluate STM capacity across a wide range of primate species and provide the first phylogenetic reconstruction of the evolution of this trait.

Our study used the infrastructure established by the ManyPrimates project - a global consortium of researchers and study sites in primate cognition research. The main goal of ManyPrimates is to approach questions about the variability and evolution of primate cognition by collaboratively building datasets that include a wide range of species and individuals. ManyPrimates has established a sustainable and long-lasting infrastructure that enables collaboration between researchers and institutions around the globe. For more details about our project, we refer to previous publications (cf. ManyPrimates et al., 2019a; 2019b; 2020; in press) and our website https://manyprimates.github.io. 
In the present study, we systematically examined the evolution of primate visuospatial STM abilities by testing 421 individuals of 41 primate species across 29 sites (see Figure 1) in a delayed response task (see also ManyPrimates et al., 2019a). The diversity of individuals and species included in our sample allowed us to evaluate how individual and species-specific factors relate to STM. In the task, an individual watched an experimenter hiding a reward under one of three cups. The individual could then retrieve the reward by choosing the correct hiding location, and we examined their relative success rates across different retention intervals (i.e. three delay conditions: $0 \mathrm{~s}, 15 \mathrm{~s}$, and $30 \mathrm{~s}$ ). This study is a continuation of our pilot study (ManyPrimates et al., 2019a), in which we validated the methodology and generated hypotheses about the phylogenetic distribution of STM abilities. However, with only 12 species studied, the pilot study did not allow us to systematically investigate the processes underlying the evolution of primate STM abilities. Here, we included this earlier data but substantially extended it by testing a much larger number of subjects from a much broader range of species. With this much larger data set, the present study alleviates this shortcoming. 


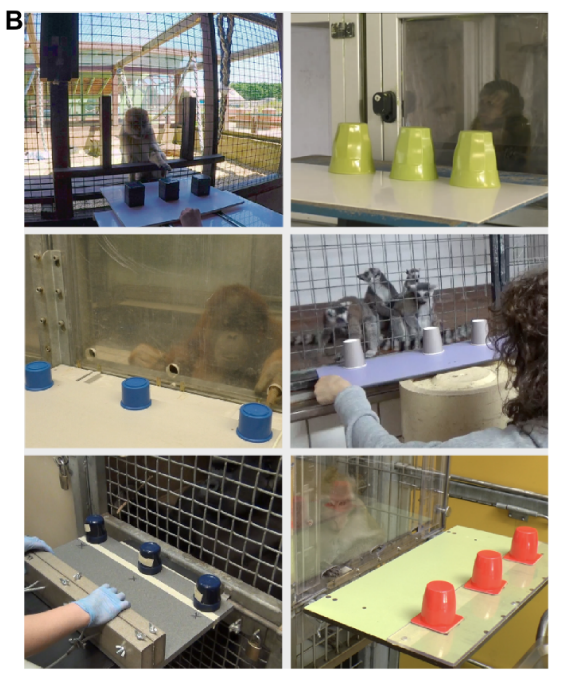

Figure 1. A) the 29 sites that contributed data to the study. B) pictures of the implementation of the delayed-response task for six species from six data collection sites (rowise starting in upper left: Macaca mulatta, Sapajus apella, Pongo abelii, Lemur catta, Gorilla gorilla gorilla, Macaca fascicularis). For a video example of the task see: https://osf.io/mntpe/.

As a first step, we evaluated the effect of delay length on STM. Based on previous work (Barth \& Call, 2006; Bartus et al., 1978) and on pilot data (ManyPrimates et al. 2019) we predicted that species would differ in their performance on the task (Harlow et al. 1932) and that longer delays would lead to decreased performance across species and taxa . In this analysis, we also tested the influence of a range of individual-level predictors on performance, such as age or experience with delayed-response tasks. Based on previous studies, we predicted that task success would decrease with age (Elmore \& Wright, 2015) whereas previous experience would lead to better task performance. In a second step, we used our experimental data to model the phylogenetic history of STM abilities. Based on pilot data and preliminary analysis (ManyPrimates et al. 2019a), we predicted that more closely related species would present more similar performance in the delayed response task. We then extend previous work by evaluating the relationship between socioecological factors and cognition (e.g. Cunningham \& Janson, 2007; Rosati, 2017; Schwartz, 2019). We tested different hypotheses about how sociality and ecology contribute to the evolution of 
short-term memory abilities in primates, over and above phylogenetic relatedness. These hypotheses were solicited from the broader research community. We circulated a description of the expected dataset via social media and mailing lists to encourage researchers in the field to submit hypotheses specifying which social and ecological variables predict STM abilities across primates. These hypotheses were collected and translated into statistical models which we then compared in a phylogenetic model comparison. This approach allowed us to be as inclusive as possible and cover a broad range of theoretical positions. The submitted hypotheses included a wide range of predictors such as diurnal-resting time, trichromacy, home range, vocal repertoire size, dietary diversity, group size, day journey length, arboreality and frugivory.

\section{Methods}

\section{Subjects}

We collected data from 421 captive primates, representing 41 different species of Platyrrhini, Strepsirrhini, Cercopithecoidea, and Hominoidea (nomenclature applied following Mittermeier et al., 2013). Subjects originated from 29 different sites located in 13 countries across the world that included zoos, sanctuaries, and laboratory facilities (Figure 1). A subset of this data has been published in ManyPrimates et al. (2019b) (see Table S1 in Supplementary Material).

We tested two families of lemurs: lemurids (true lemurs and relatives, 8 species, $n=72$ ) and indriids (sifakas and relatives, 1 species, $n=9$ ). We included three families of Platyrrhini: cebids (capuchin and squirrel monkeys, 3 species, $\mathrm{n}=92$ ), callitrichids (marmosets and tamarins, 4 species, $\mathrm{n}=50$ ) and atelids (spider monkeys and relatives, 2 species, $\mathrm{n}=4$ ). The majority of species and individuals studied represented the Cercopithecidae clade (Cercopithecidae). We studied both 
subfamilies of this group: the generalist cercopithecines (cheek-pouched monkeys, 10 species, $\mathrm{n}=$ 93 (including 2 hybrid subjects, $P$. anubis $\mathrm{x}$ cynocephalus)) and the folivorous colobines (leafeating monkeys, 3 species, $n=13$ ). Finally, both families of apes were tested: the hylobatids (gibbons, 5 species, $\mathrm{n}=9$ ) and the hominids (great apes, 5 species, $\mathrm{n}=79$ ).

Our sample thus covers the majority of radiations within the primate order. However, Tarsiiformes (tarsiers) and Lorisiformes (lorises, galagos, and relatives) were not studied due to a lack of participating sites offering access to these animals. Several families of Platyrrhini (Aotidae and Pitheciidae) and lemurs (Cheirogaleidae, Daubentoniidae, Lepilemuridae) were not included for the same reason.

Experiments and participating institutions complied with the ethics guidelines of the ManyPrimates project (https://manyprimates.github.io/ethics/) and explicit ethical approval was obtained from each participating institution. In the Supplementary Material we provide a detailed description of each data collection site including housing and research practices and the procedure that was used to obtain ethical approval. A short video of the task with different species from different sites can be found here: https://osf.io/mntpe/.

\section{Materials}

We tested all individuals in the delayed-response task. This paradigm was chosen given its internal validity, simplicity and popularity in the animal cognition literature. The general setup comprised a rectangular board and three identical, opaque cups. High-value food items were used as rewards 
(Figure 1B; the size and food type varied across species and sites). In the test, the board was placed in front of the subject, outside the enclosure. The cups were evenly spaced on the board with at least $10 \mathrm{~cm}$ between them (center to center). The following aspects of the setup varied between sites: board size, cup size and color, distance between cups, food reward used, testing arrangements (group vs. individual testing), and subject experience with object choice tasks (task experience also varied between subjects at the same site). Some of these differences were due to differences between testing facilities (e.g., the testing arrangements), others due to differences between primate species or cohorts (e.g., the type and size of the food rewards). We documented these differences in the Supplementary Material (see Table S1) and considered some of these differences as predictors in our confirmatory analysis (board size, cup distance, task experience).

In our pilot study (ManyPrimates et al., 2019a), some aspects of the setup systematically co-varied with species (board size and cup distance). We therefore asked institutions to vary board size and cup distance independent of species body size (but given that we included our pilot data we could not completely remove this confound in this way). To ensure the proper implementation of the setup and the procedure, each site recorded a short video of the test setup and implementation of a trial prior to actual testing. The project coordinators checked the videos and provided feedback if they noticed any deviations from the protocol.

\section{Design}

The main experimental (within-subject) manipulation was the time passed between hiding a food item in full view of the subject and allowing the subject to retrieve it. The intervals were $0 \mathrm{~s}, 15 \mathrm{~s}$ and 30 s resulting in three delay conditions: short, medium and long. 


\section{Procedure}

Depending on the site, the subjects were tested individually or in group settings. In the case of group settings, researchers specified the focal individual ahead of time and distracted non-focal individuals during testing by giving them access to additional food or enrichment items. Before participating in the test, some subjects with little object-choice experience received additional training to ensure reliable choice behaviors. They were trained to reliably point to or reach for fully visible food items placed on the board before the start of the experiment. In our choice training protocol, subjects were presented first just with an out-of-reach food item, then with a food item that was covered by an opaque cup. Finally, they had to choose between two cups, one visibly baited and the other one not. Only when subjects made reliable, unambiguous choices in each training phase were they presented with the test (but there was no formal progression criterion).

At the beginning of a trial, the experimenter (E; familiarity between $\mathrm{E}$ and the subjects varied between sites) pulled the board back so that the subject could not reach it. E stood or sat behind the board and placed the three cups next to each other with the opening facing the subject. Next, E showed the subject a food item and placed it in front of one of the cups. Then E put the cups down one by one, thereby hiding the food item, always starting with the cup on the left from E's perspective. Depending on the condition, E waited either $0 \mathrm{~s}$ (short delay), $15 \mathrm{~s}$ (medium delay) or $30 \mathrm{~s}$ (long delay) before pushing the board towards the subject. The delay started once E had put down the last cup (on the right). While pushing the board forward, E looked down and center to avoid inadvertent cueing. The subject made a choice by either pointing to or touching one of the cups. If the subject chose more than one cup simultaneously, E pulled the board back and pushed it forward again to ensure an unambiguous choice. If the subject did not make a choice within 60 $\mathrm{s}$, the entire trial (including hiding and delay) was repeated. After the subject chose a cup, E pulled 
the board back and lifted the indicated cup. If the cup revealed the food item, the subject got it as a reward. If the cup did not cover the food item, the subject got no reward. After E had lifted the indicated cup (and passed the reward to the subject in case of a correct choice), E turned over the remaining two cups with the open side facing to the subject and, in case of an incorrect choice, took the remaining food reward back in preparation for the next trial. The same food item was used again in the next trial. Sessions were terminated if a subject did not make a choice in three consecutive trials. Further, data collection was stopped with a subject if three sessions had to be terminated because the subject did not make a choice.

For each subject, the hiding location was pseudo-randomized across trials with the constraints that the same location occurred no more than two times in a row and that each hiding location occurred an equal number of times per condition. Trials were grouped in blocks, with each block comprising three trials of the same condition (either short, medium, or long delay). Each set of nine trials comprised three blocks, one per condition. The order of conditions across the three blocks was randomized. Different subjects received different randomizations.

The number of trials per test day (session) varied between sites, species and individuals, with the constraint that there were at least three trials (one block) per test day. To be included in the analysis, individuals had to contribute a minimum of 9 trials and a maximum of 36 trials, equally distributed across conditions.

\section{Scoring}

We scored whether the subjects chose the correct cup, i.e. whether they manually indicated the location in which the food item was hidden. This resulted in a binary (0: incorrect, 1: correct) variable for each trial. 


\section{Analysis}

The confirmatory and phylogenetic analyses were pre-registered before we started to inspect the data (https://osf.io/sf3bx). All data and analysis scripts are available in a public repository (https://github.com/ManyPrimates/mp1_short_term_memory). All statistical analyses were performed in $\mathrm{R}$ version 4 .0.3 (R Core Team, 2020).

For the comparison to chance level we aggregated the data for each individual in each of the three delay conditions and used the function ttestBF from the package BayesFactor (Morey \& Rouder, 2018) to compute the Bayes Factor (based on a Bayesian t-test) in favor of the hypothesis that the average proportion of correct responses in a condition was above 0.33 .

All other models were fitted to the trial-by-trial data as Bayesian generalized linear mixed models (GLMM) with a logit link using the function brm from the package brms (Bürkner, 2017). Model parameters for each model were estimated by collecting 10000 samples from eight independent MCMC chains, removing the first 5,000 samples for burn-in. Priors for all models are reported in the Supplementary Material. All models converged without problems with Rhat values $<1.01$. The model outputs can be accessed via the online repository.

We computed WAIC (widely applicable information criterion) scores for every model, and for the model comparison, we also added WAIC weights. Following McElreath (2018), we used these metrics to rank models. In addition, we inspected the $95 \%$ Credible Intervals for the test predictors of interest. 
The confirmatory model had the following structure:

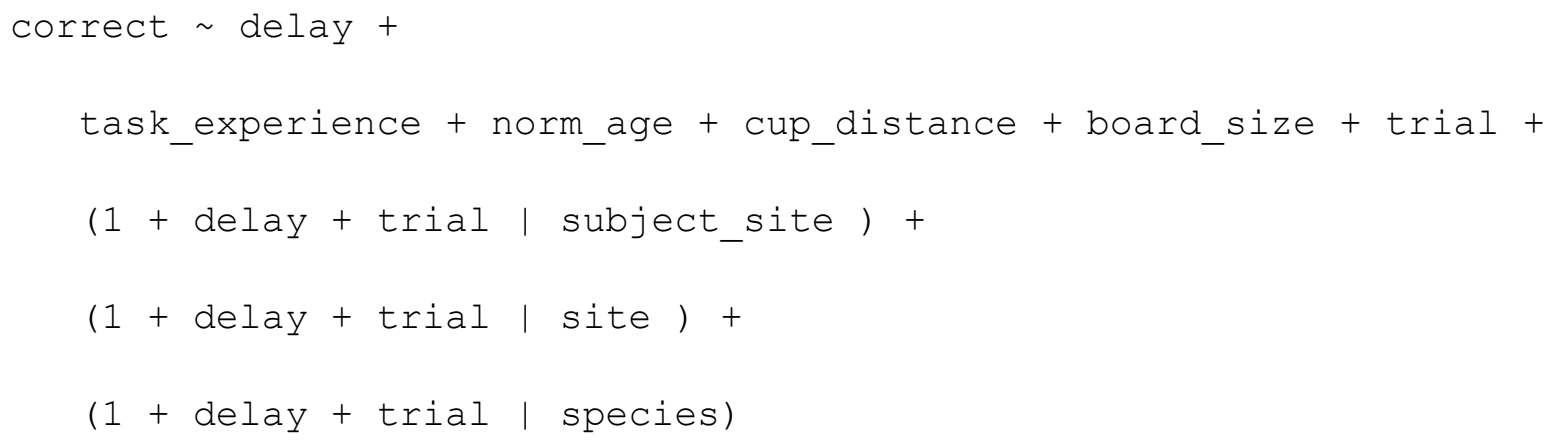

where correct noted whether an individual chose the correct cup on a given trial. Delay was the length of the time between hiding the food and choosing a cup. For the confirmatory analysis, this was coded as a three-level factor (centered at medium delay of $15 \mathrm{~s}$ ). For the phylogenetic analyses, it was coded as a two-level factor (medium and long delay coded as 0 and short delay coded as 1). This factor coding is different from the numeric coding of delay we pre-registered. In the Supplementary Material, we explain why we deviate from our pre-registration here. Task_experience noted whether the individual has participated in comparable object-choice studies before (coded as yes/no). Norm_age was the individual's age normalized by the maximum recorded life span of that species. Cup_distance was the distance between the cups and board_size the width of the board on which cups were presented (both in $\mathrm{cm}$ ). Trial noted the trial continuously across sessions. Subject_site was a unique identifier for each subject. Site was the data collection site and species noted the individual's species. All numerical predictors were scaled to have a mean of 0 and a standard deviation of 1 . The alternative model in the confirmatory analysis had the same structure except that it did not include delay as a predictor.

The phylogenetic baseline model had the following structure:

correct $\sim$ delay + 


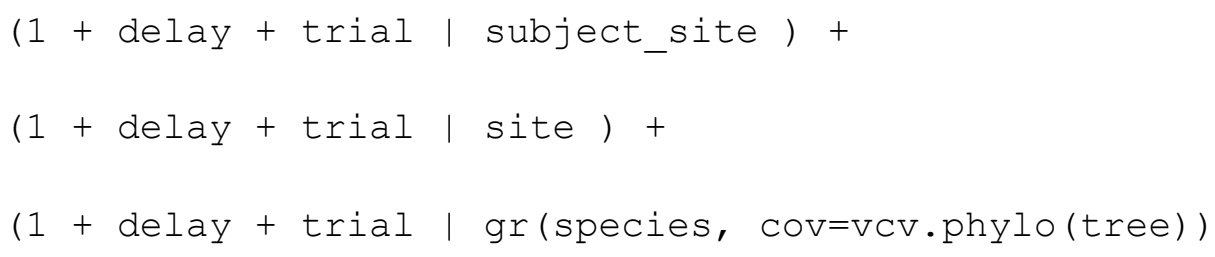

Here, tree is a consensus primate phylogeny from the 10ktrees project (Arnold et al., 2010), pruned to include only those species included in our study. The $\mathrm{VCV} \cdot \mathrm{ph} Y \mathrm{l} \circ$ function of the R package ape (Paradis \& Schliep, 2019) was used to compute the expected correlations between the tip values (i.e. per-species values) of a trait evolving along this tree according to a Gaussian process model of evolution (including the Brownian motion model). The resulting matrix is used as the covariance matrix for all random effects of species.

In contrast to the confirmatory model, the phylogenetic models did not include the control predictors board_size, cup_distance, task_experience, and trial. The reasons were: a) some of these variables were confounded with phylogeny (e.g. board_size and phylogeny both co-varied with body size), b) we had no hypothesis about if and how they interact with the test predictors, and c) excluding them facilitated the interpretation of the influence that test predictors had on performance.

To assess the degree to which performance in the task follows a pattern expected by the phylogenetic relatedness between species, we compared the phylogenetic baseline model to an identical model that made no assumptions about the correlations between species' performances (i.e. random effect for species: ( $1+$ delay + trial | species)). We also quantified the phylogenetic signal in the data: Following a systematic comparison of indices (Münkemüller et al., 2012), we report Blomberg's $K$ and Pagel's $\lambda$ (Blomberg et al., 2003). The first of these (K) 
can be understood as the ratio between the observed degree of variation in the value of some variable across the tree about its mean and the expected degree of variation under a Brownian motion (BM) model of evolution for that variable; the latter $(\lambda)$ is instead a scaling factor which, applied to the internal branch lengths of a tree, maximizes the likelihood of the data under the same BM model - values below 1 shrink branches, effectively reducing the effect of deep history. Despite being different indices, Blomberg's $K$ and Pagel's $\lambda$ share some key characteristics: their lower bound is zero (i.e., phylogenetic independence), and more substantial deviations from zero indicate stronger relations between per-species performance and phylogeny (i.e., a Brownian motion model of trait evolution). Values close to one suggest that per-species performance is distributed as expected by phylogeny. We computed Blomberg's $K$ and Pagel's $\lambda$ for the predictions of our phylogenetic baseline model (see below). We escaped the circularity of measuring the phylogenetic signal in a model which explicitly takes phylogeny into account via a partial constraint: while the estimated per-species random effects reflect phylogeny in their correlations, the actual size of these effects relative to either the fixed effects or other non-phylogenetic random effects (such as those of site or individual) is not strongly constrained and depends on the data. Thus, the phylogenetic signal in the overall model output can in principle be arbitrarily low if this is what fits the data well.

The phylogenetic model also accounted for the repeated testing of individuals and the nesting of individuals within sites. Thus, in order to get per-species estimates of the signal, we repeatedly sampled one random individual and one random site to represent each species. For each of these samples, we computed the phylogenetic signal in the linear predictor for the 3 possible values of 
delay. We collected 3,333 samples of individuals and sites, resulting in $\sim 10,000$ calculations of the phylogenetic signal in total, yielding the distribution described below.

To test which social and ecological species-level characteristics relate to STM performance, we conducted a phylogenetic model comparison. Given the myriad of species-level characteristics to consider, any particular selection largely hinges upon one's theoretical views concerning which social or ecological factors drive cognitive evolution. ManyPrimates, as well as the broader research community, is composed of scientists with a wide range of theoretical perspectives. To do justice to this plurality, we decided to solicit theories from the research community as part of a "modeling challenge". In February of 2020, before data collection had been completed, we circulated a rough description of the expected dataset via social media (Twitter) and mailing lists (International Primatological Society, Cognitive Science Society) and asked researchers to submit theories nominating the species-level characteristics they deemed most predictive of primate STM. Theories were restricted to include only characteristics external to animals; that is, characteristics that reflect a species' social or ecological environment. We deliberately excluded commonly used internal predictors of cognitive performance - e.g., brain size - because they do not specify any external pressures that require adapting to and therefore provide no answer to the question of why a given species evolved an ability.

In addition to detailing their model, we also asked researchers to submit sources for the data on the species-level predictors in their model. For many submissions, this information was missing or incomplete and we had to search for it ourselves. We did not find sources for all predictors for all species. As a consequence, we could not include all submitted models in the model comparison. 
We did not find data for many predictors for two species (Allen's swamp monkey (Allenopithecus nigroviridis) and Hamlyn's monkey (Cercopithecus hamlyni)), and so we decided to exclude data from these species from the phylogenetic analysis.

We would also like to note that the basis for some species-level predictor variables was very sparse. In many cases, we had to use secondary sources because the primary source was inaccessible. At other times, multiple sources were available but yielded substantially different estimates of species-level predictors. We used the following steps to decide which source to use in case we had more than one. First, we prioritized the source that provided data for the most species (to ensure comparability across species). To fill the remaining gaps, we used the source that provided the next most data for the remaining species, iteratively proceeding until we were no longer able to obtain further multi-species estimates. Finally, we used sources for individual species with the largest reported sample size to fill any remaining gaps. The associated online repository contains the final spreadsheet with the data and the sources we used for our analyses. We believe our repository provides the best possible estimates of these predictors given available data. At the same time, by making our dataset publicly available, we welcome refinement of our estimates via future studies and/or unpublished data.

The models that entered the phylogenetic model comparison were constructed by adding the submitted predictor variables to the phylogenetic baseline model as a main effect and a fixed effect interaction with delay (centered at $15 \mathrm{~s}$ ). For example, one submission suggested that group size was related to short-term memory performance. The fixed effects structure of the corresponding model was therefore group_size * delay. We compared a total of 10 distinct models, 
including the baseline model. Table 1 lists all the individual predictors and Table 2 the resulting models.

Table 1: Predictors used to infer STM evolution in primates.

\begin{tabular}{lll} 
Predictor & Definition & Variable type \\
\hline body_size & Average female body mass of a species (kg). & numeric \\
\hline color_vision & $\begin{array}{l}\text { Species' capacity to differentiate colors. Levels } \\
\text { were dichromatic, trichromatic or polymorphic } \\
\text { (i.e. both dichromatic and trichromatic individuals } \\
\text { are present in a population). }\end{array}$ & factor (three levels) \\
\hline day_journey_length & Daily traveled distance (in m) & numeric \\
\hline diet_diversity & $\begin{array}{l}\text { Trophic guild a species belongs to (e.g., folivore, } \\
\text { frugivore, omnivore) }\end{array}$ & factor (six levels) \\
\hline dietary_breadth & $\begin{array}{l}\text { Number of dietary categories eaten by each } \\
\text { species. Categories were defined as vertebrate, } \\
\text { invertebrate, fruit, flowers/nectar/pollen, } \\
\text { leaves/branches/bark, seeds, grass and } \\
\text { roots/tubers. }\end{array}$ & numeric \\
\hline feeding_budget & $\begin{array}{l}\text { Percentage of time spent feeding from the total } \\
\text { activity budget of a species. }\end{array}$ & numeric \\
\hline group_size & Average number of individuals in social group & numeric \\
\hline home_range & Average home range size (in ha). & numeric \\
\hline percent_frugivory & Percentage of fruit in a species' diet. & numeric \\
\hline diurnal_resting_ti & Percentage of time spent resting during the day & numeric \\
\hline me & Primary habitat (levels: arboreal or terrestrial) & factor (two levels) \\
\hline terrestriality & $\begin{array}{l}\text { Number of distinct calls in a species' vocal } \\
\text { repertoire }\end{array}$ & numeric \\
\hline vocal_repertoire & & \\
\hline & &
\end{tabular}

Note: Data sources for the different predictors are listed in the spreadsheet in the associated online repository.

As part of the exploratory analysis, we fit additional main effects models for predictors submitted in models in which they were combined with others. For example, one submission included the 
term percent_frugivory * terrestriality. From this, we constructed two additional models, one for percent_frugivory and the other for terrestriality. The results of this analysis are reported in the Supplementary Material.

In the Supplementary Material, we provide a number of additional analyses. First, we report a prior-sensitivity analysis for the phylogenetic models in which we constrained the influence of phylogeny on the model by reducing the variation between per-species random effects. We also report the results of several exploratory analyses, including an assessment of how variable specieslevel performance was across data collection sites, an assessment of the task's split-half reliability (which was acceptable at $r=0.62$ ) as well as additional variants of the confirmatory and phylogenetic models.

\section{Results}

\section{Descriptives}

As a group, the primates we tested successfully remembered where food was hidden in the delayedresponse task. When averaging across all individuals and species, the proportion of correct responses was higher than a level expected by random guessing (33\% correct) in all conditions; $m_{\text {short }}=0.76\left(s d=0.24\right.$; Bayes Factor $\left(\mathrm{BF}_{10}\right)$ in favor of the hypothesis that performance was above $\left.0.33=4.2 * 10^{112}\right) ; m_{\text {medium }}=0.55\left(s d=0.25 ; \mathrm{BF}_{10}=2.7 * 10^{50}\right) ; m_{\text {long }}=0.51\left(s d=0.24 ; \mathrm{BF}_{10}=\right.$ $6.5 * 10^{37}$ ); see also Figure 2. When comparing across species, performance levels appeared to be clustered within clades and along phylogenetic lines: Hominoidea had a higher proportion of correct responses compared to Cercopithecoidea, who in turn performed above Platyrrhini and Strepsirrhini (Figure 2). However, performance varied greatly between individuals, such that 
within each delay condition, the response distribution for a given species overlapped with that of most other species.
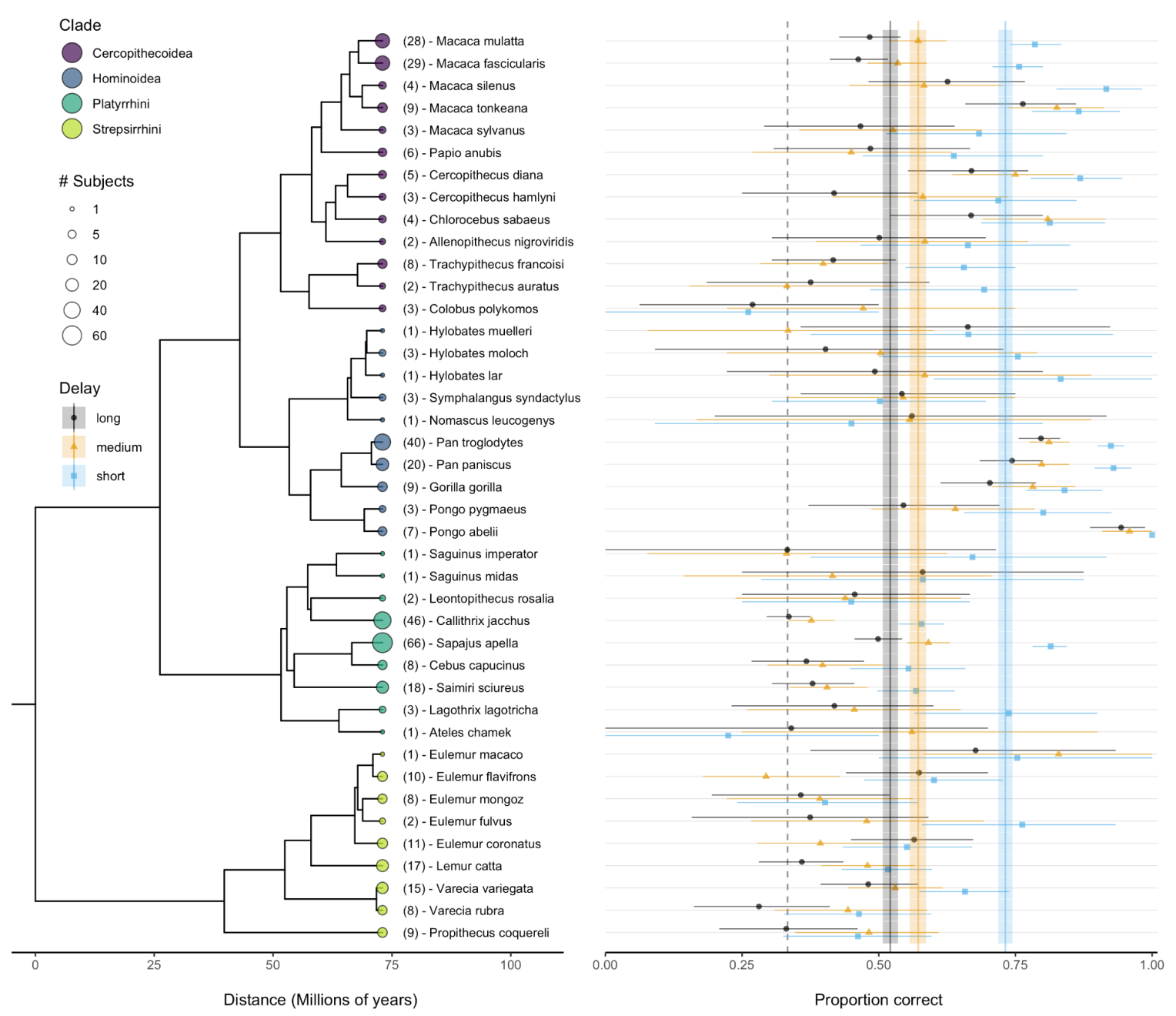

Figure 2. Chronogram (left) and performance in the delayed response task (right) for the 41 primate species tested. Phylogenetic data were obtained from 10kTrees (Arnold et al., 2010). Branch lengths are proportional to absolute time. The size of the filled points is proportional to the number of subjects for each species. Colored shapes (with 95\% CI) show the mean performance per species in the three delay conditions. The colored vertical lines show the mean performance across species (with $95 \% \mathrm{CI}$ ) in the three delay conditions. The dotted vertical line shows a level of performance expected by chance ( $33 \%$ correct).

\section{Confirmatory analysis}


Our confirmatory analysis tested if delay length (i.e. retention interval) affected primates' performance in the delayed response task. To assess the effect of delay length, we compared a full model that included delay as a predictor to a model lacking it. A Bayesian model comparison based on WAIC (widely applicable information criterion) scores and weights clearly favored the model including delay (delay: WAIC $=14205.50$, se $=88.47$, weight $=1.00$; no delay: WAIC $=14786.99$, $\mathrm{se}=80.52$, weight $=0.00)$. Compared to the medium $(15 \mathrm{~s})$ delay condition, performance was better in the short $(0 \mathrm{~s})$ delay condition $(\beta=0.90,95 \%$ credible interval $(\mathrm{CrI}): 0.67-1.13)$ and worse in the long ( $30 \mathrm{~s})$ delay condition $(\beta=-0.25,95 \% \mathrm{CrI}:-0.36--0.12)$. Thus, performance in the delayed response task decreased when the delay between hiding the food and retrieving it increased, replicating the results of previous studies (Barth \& Call, 2006; ManyPrimates et al,. 2019a) and extending it to 29 new species (see Supplementary Material Table S1).

The confirmatory model also included a range of control predictors, which reflected variability between individuals and the physical implementation of the task. Figure 3 shows that individuals with experience in delayed response tasks performed better than individuals naïve to such tasks ( $\beta$ $=0.25 ; 95 \%$ CrI: $0.01-0.49$ ). Individuals who were tested with cups further apart were also more likely to perform better ( $\beta=0.48 ; 95 \% \mathrm{CrI}: 0.26-0.71)$. The latter result is likely to be confounded with body size (and phylogeny); larger individuals (mostly great apes) were tested with cups placed further apart (see Supplementary Material). 


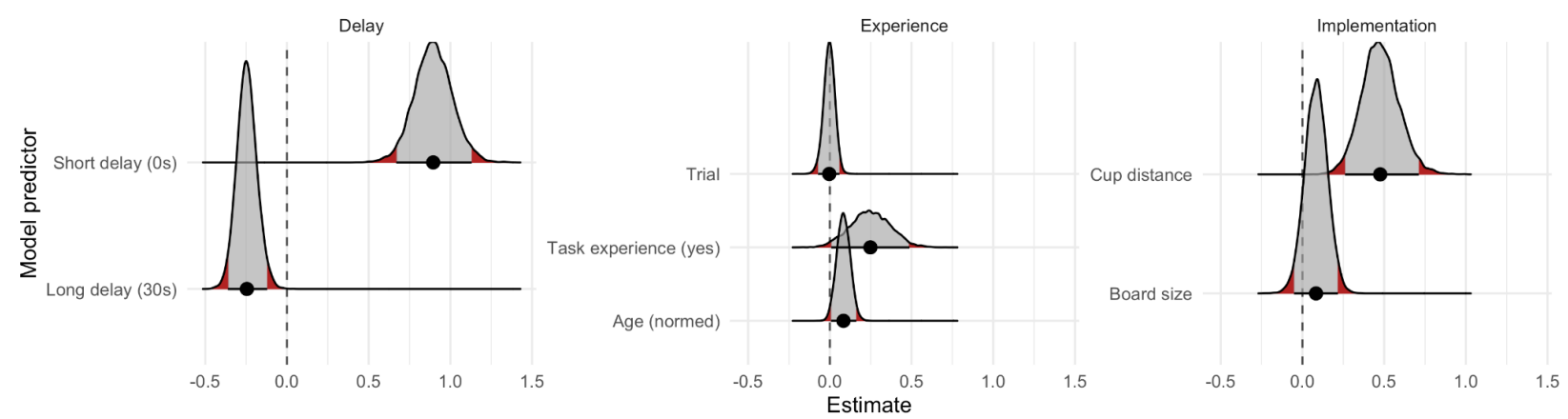

Figure 3. Posterior distributions for predictors in the confirmatory analysis. Gray regions (and error bars) show 95\% CrIs.

Performance increased with age (normalized by the maximal recorded lifespan for the species; $\beta$ $=0.08 ; 95 \% \mathrm{CrI}: 0.01-0.16)$, contradicting our pre-registered prediction that STM abilities would decline with age. In the Supplementary Material, we also report a model that posits a quadratic relation between age and performance to explore the possibility that performance increases early in life but then drops off again in old age. However, adding this nonlinear term did not improve the fit of the model $^{1}$.

\section{Phylogenetic analyses}

Through a set of phylogenetic analyses, we tested whether performance in the delayed response task was linked to phylogeny and species-level predictors. The species in our sample differed in the amount of evolutionary history they shared; more closely related species might have more similar STM abilities because they evolved from a common ancestor. The confirmatory analyses

\footnotetext{
${ }^{1}$ We did not pre-register the inclusion of testing situation (group vs. individual) as a predictor. This was because we used the same confirmatory model as for the pilot study, which did not include this predictor due to a lack of variation in the data. In a model that includes testing situation as a predictor in the confirmatory model, we find no difference between testing situations ( $\beta=-0.03,95 \% \mathrm{CrI}$ : $-0.30-0.25)$ while all other predictor estimates remain unchanged.
} 
reported above did not account for this potential source of structure in our data. As noted in the Methods section, for our phylogenetic analyses, we constrained the covariance between specieslevel effects to reflect the expected correlations between species based on a Brownian motion model of evolution applied to a consensus primate phylogeny (Arnold et al., 2010). When compared to a model that made no assumptions about the covariance between species, a phylogenetic model provided a much better fit to the data (phylogeny: WAIC $=14091.57$, se $=$ 87.58 , weight $=0.87$; no phylogeny: WAIC $=14095.37$, se $=87.53$, weight $=0.13$ ). This result suggests that species differences in performance in the delayed response task map onto phylogenetic relatedness between species (Figure 4).

\section{Phylogenetic signal}

This result was also reflected in the indices quantifying the phylogenetic signal. For Blomberg's $K$ the overall mean was 0.33 (95\% HDI: $0.17-0.52$ ) and for Pagel's $\lambda$ the overall mean was 0.70 (95\% HDI: $0.41-0.99)$. Thus, in both cases, the phylogenetic signal was measured to be reliably different from zero, suggesting a considerable overlap between the distribution of performance in the delayed-response task and species phylogeny. 

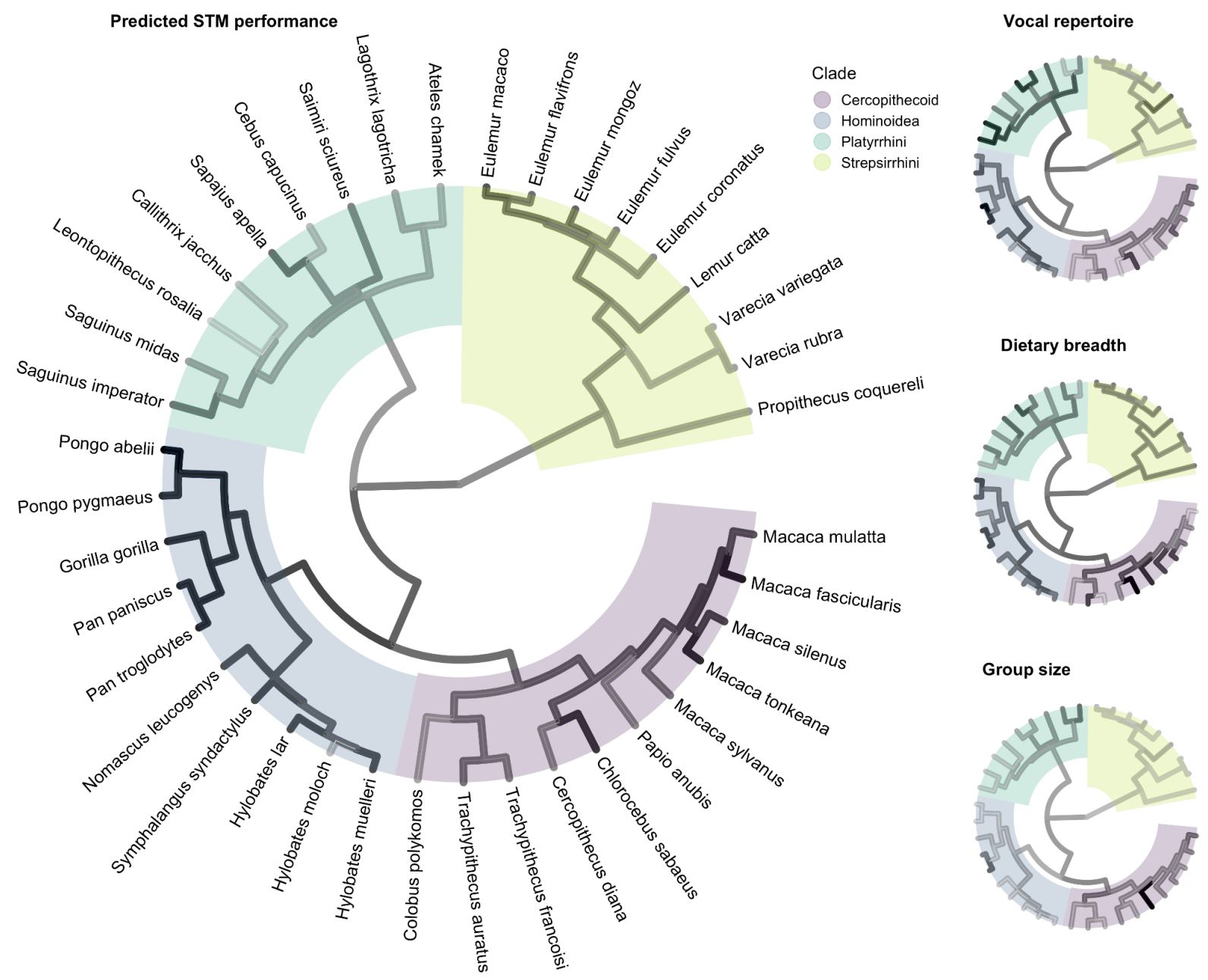

Figure 4.Phylogenetic tree with ancestral state reconstruction based on a Brownian motion model of trait evolution for STM performance (left) and the predictors of the three highest ranking models in the model comparison (right). For STM, darker lines correspond to higher probabilities (as predicted by the model) of choosing the correct cup in delay trials. For the predictors, darkness of the lines corresponds to the estimated predictor value, with darker lines corresponding to higher values (the scale differs across variables). Branch lengths are proportional to absolute time. Shading highlights the different clades.

The substantial phylogenetic signal in our dataset indicates that closely related species have more similar STM abilities. Still, phylogenetic relatedness cannot fully explain why some species have evolved more potent STM skills than others, as relatedness does not explain what non-genetic (e.g. environmental or social) factors have exerted selective pressure on different species. Thus, in the 
next analysis, we examined which species-level social or ecological characteristics were associated with STM abilities over and above phylogeny.

\section{Phylogenetic model comparison}

In the phylogenetic model comparison, we compared predictor models that specified one or more species-level, external variables that (presumably) relate to performance to a baseline mode that only accounted for phylogeny. Surprisingly, none of the predictor models outperformed the baseline model. All predictor models had WAIC scores higher than the baseline model and thus lower relative weight (see Table 2). However, the minute differences in WAIC scores and weights between the baseline, the vocal repertoire, and the dietary breadth model showed that these models were basically indistinguishable from one another. Because the baseline model offered a more parsimonious explanation of the data, we nonetheless conclude that the additional species-level predictors contributed little to explaining differences between species over and above phylogeny. In other words, knowing, for example, a species' vocal repertoire provides little additional information about STM abilities when already knowing its position in the phylogenetic tree.

The distribution of the predictor variables across species may explain this result. We computed the phylogenetic signal in our predictor variables and found many to be strongly aligned with phylogeny (see Table S4 in the Supplementary Material). Figure 4 uses ancestral state reconstruction to visualize the phylogenetic distribution of STM performance as well as the predictor variables from the three highest-ranking models. While many of the predictor variables were positively correlated with STM abilities when ignoring phylogeny (see Figure S4 Supplementary Material), once accounting for phylogeny, these variables provide little additional 
information about species' STM abilities. Our results thus suggest that observed patterns arise because closely related species tend to live in more similar environments that share certain social and ecological features, rather than environmental features - at least the ones we investigated independently affecting the evolution of STM abilities.

Table 2: Phylogenetic model comparison.

\begin{tabular}{lccc} 
Model (predictors) & WAIC & SE & weight \\
\hline (baseline) & 14091.57 & 87.58 & 0.20 \\
\hline vocal_repertoire & 14091.70 & 87.66 & 0.19 \\
\hline dietary_breadth & 14092.19 & 87.67 & 0.15 \\
\hline group_size & 14092.83 & 87.68 & 0.11 \\
\hline home_range/body_size + feeding_budget $*$ dietary_breadth & 14093.14 & 87.81 & 0.09 \\
\hline diurnal_resting_time & 14093.39 & 87.67 & 0.08 \\
\hline percent_frugivory $*$ terrestriality & 14093.83 & 87.79 & 0.07 \\
\hline color_vision & 14094.19 & 87.72 & 0.06 \\
\hline day_journey_length * group_size & 14095.12 & 87.85 & 0.03 \\
\hline diet_diversity & 14096.46 & 87.93 & 0.02
\end{tabular}

Note: All models had the same basic structure (baseline model): correct delay $+(1+$ delay + trial | subject_site + site + species) with species capturing phylogeny following a Brownian Motion model of trait evolution (see main text for details). The predictors in the table were added to this baseline model as interactions with delay.

\section{Discussion}

In this study we compared short-term memory (STM) abilities across the largest and most diverse primate sample used in an experimental study to date. Primates from all clades performed above 
chance in at least one of the delay conditions, so that STM could be regarded as a basal cognitive trait of primates. Across species, we also found that the longer the delay between hiding and retrieving food rewards, the worse the performance on memorizing the location of the food. This finding confirmed our predictions and replicated the results of previous tests of nonhuman primates using the delay-response task (Barth \& Call, 2006). A similar delay effect is also found in human studies of non-verbal auditory memory, and is interpreted as reflecting the construal of STM as a transient storage of limited capacity (e.g., Mercer \& Mckeown, 2014).

The primates' performance was further mediated by phylogeny, age and experience. Individuals with prior experience in such delayed response tasks performed better in this task than naïve individuals, and performance increased with relative age. A similar age effect has also been reported in human children, whose performance in a two-cup delayed-response task with increasingly long delays was positively predicted by the children's age (Diamond \& Doar, 1989). Phylogenetic analyses revealed that species differences can be predicted by their phylogenetic relatedness. That is, closely related species performed more similarly in the delayed-response task compared to more distantly related species.

Within the phylogenetic tree, we found higher performance by individuals in the branch of Hominoidea compared to Cercopithecoidea, who in turn performed above Platyrrhini and Strepsirrhini. This pattern was not absolute in that, for example, some Cercopithecoidea (e.g., Macaca tonkeana) performed comparably to the Hominidae (e.g. Gorilla gorilla), while others (e.g. Trachypithecus francoisi) performed more similarly to some of the Strepsirrhini (e.g., Lemur catta). Additionally, performance varied greatly between individuals, such that the response distribution for a given species overlapped that of most other species within each delay condition, 
reflecting previous research in macaques and gorillas that has highlighted inter-individual variation in performance in tests of memory and learning (e.g., Altschul et al., 2016; Egelkamp et al., 2019).

Overall, however, the pattern of results we observed suggests systematic variation across species. This opens up the question of which selective pressures are responsible for the pattern we observed. We approached this question in our phylogenetic model comparison. The different models represent a range of hypotheses about which social or ecological variables may influence STM abilities. The models test the assumption that if one were to - hypothetically- intervene on the respective predictor variable(s), the STM abilities of a species should change over time irrespective of the species' location in the phylogenetic tree. It is important to note that this approach is not able to detect effects of predictors if they are confounded with phylogenetic relatedness. However, this clustering would suggest that the predictor did not have a causal effect in and of itself, but rather that the effect was conditional on other factors that are only present in certain parts of the phylogenetic tree. From a statistical point of view, we were thus looking for predictors that explained species differences once phylogenetic relatedness had been accounted for.

Contrary to our predictions, we found no evidence that any of the predictor variable(s) we considered had such a general effect. None of the models that included a predictor substantially outperformed a baseline model which only accounted for phylogeny. That is, when knowing a species' location in the phylogenetic tree, learning about its social or ecological characteristics (e.g. vocal repertoire or dietary breadth) did not substantially improve the ability to predict that species' STM capacity. Thus, on the one hand, the strong phylogenetic signal suggests some 
systematic pressures working on the evolution of STM abilities, but on the other hand, we did not identify these pressures in our phylogenetic model comparison. This results contrasts with previous research that has found links between primate brain size (as a proxy for cognitive abilities) and some of the predictors also included here (e.g., diet and home range, DeCasien et al., 2017; Powell et al., 2017)

There are a number of (mutually non-exclusive) reasons why the predictors we considered did not contribute to explaining the phylogenetic distribution of STM abilities. First, the predictors that were submitted as part of the modeling challenge could truly have no influence and other specieslevel social or ecological variables need to be considered. In a similar way, other cognitive abilities (such as other executive functions, like control of attention; Morey \& Bieler, 2013) could be responsible for the differences across species in performance in the delayed-response task. Such a scenario assumes that variation in STM abilities in primates are essentially a product of other evolutionary processes which would shift the locus of explanation to identifying which variables predict performance in other cognitive abilities. Second, the effect of the predictor variables might not be as general as assumed in the models. For example, dietary breadth might have an effect on STM abilities only when considered in conjunction with home range size. Third, the predictors might be measured at the wrong time and for the wrong species. We estimated the predictors based on data from extant species, which leaves open the possibility that they might have been different at the time in which they exerted a selective pressure on ancestral species. Finally, the measurement quality of the predictors could be insufficient. In fact, in many cases, we had to rely on secondary sources to find values for some predictors. Thus, it might be that our predictor values do not accurately represent species' sociality or ecology. If this were the case, however, it would also cast 
doubt on previous work because we relied on published studies for most of our predictor values. Other reasons are of course conceivable. All of this shows that there is still much work to be done when it comes to identifying the selective pressures that shaped the evolution of STM. Nevertheless, the dataset we collected here provides a crucial resource to tackle this problem.

The results of the present study also inform the debate around the neural mechanisms of STM. The dorso-lateral prefrontal cortex has been suggested to be a 'necessary and sufficient' substrate of STM (as suggested by, e.g., Riley \& Constantinidis, 2016). However, the successful performance of some lemurs in the present study, and the absence of a granular dorso-lateral prefrontal cortex in strepsirrhine species (Wise, 2017), speak against this hypothesis. The emergence of a dorsolateral prefrontal cortex in anthropoids may have contributed to a more robust STM through increased executive control (consistent with, e.g., Postle, 2015). Consequently, STM efficiency would be further boosted in primates endowed with a more complex dorso-lateral prefrontal cortex, enabling individuals to withstand interference or mere time-based decay and, thus, to hold items in memory for longer periods of time. More research using additional STM tasks of various complexity and modalities are needed to adjudicate between these various possibilities.

An interesting extension to the current work would be to examine working memory across species using the ManyPrimates approach. Whilst STM refers to the passive retention of information over the short-term, working memory is a multi-component system responsible for the active maintenance and manipulation of information (Cowan, 2008; see Basile \& Hampton, 2013, for discussion of the differentiation between STM and working memory in non-human primates). Although models differ in their explanation of working memory, all share the 
requirement of attentional control to maintain and manipulate relevant information and to shield the system from distraction and interference during retention (Burgoyne \& Engle, 2020; Cowan, 2008; Engle, 2018; Oberauer, 2009). Working memory is also essential for the maintenance and manipulation of abstract rules in both humans and non-human primates (Bunge, 2004; Mansouri et al., 2020; Nakahara et al., 2002). Such abstract rules underpin many higher-order cognitive abilities; indeed, unlike STM, working memory has been shown to be related to measures of general intelligence and executive functioning in humans (Conway et al., 2003; Engle, 2018; Miyake et al., 2000; Unsworth \& Engle, 2007). As such, phylogenetic analysis of working memory in non-human primates would prove valuable to our understanding of the evolution of cognitive processes thought essential for goal-directed behavior. Some simple extensions to our current design to ensure the recruitment of working memory (cf. STM) processes could be to fill the retention interval of our memory paradigm with a distraction task, utilize a delayed match-tosample task with distraction (e.g., Basile \& Hampton, 2013), or utilize a version of the selfordered search task (e.g., Petrides, 1995).

The results of our phylogenetic analysis are of course conditional on the sample we tested. Despite a concerted effort to obtain a large, diverse sample of primates, data collection for the present study was nevertheless influenced by the relative abundance of some species of primates over others in captivity, and our relative ability to test them. Certain taxa such as chimpanzees, capuchin monkeys, squirrel monkeys, and long-tailed macaques were tested more frequently because they are common in research facilities and zoological institutions and are typically housed in large groups. Other taxa, for instance, pair-living gibbons or tamarins, had much smaller sample sizes (some as low as only one individual per species). To be more fully representative of the primate 
order, future research may have to expend additional efforts to recruit understudied species. Most importantly, to increase representativeness, future research should aim to also study primates living in the wild instead of captive settings. Testing wild individuals and comparing their performance with the results presented here would allow us to evaluate the extent of a potential captivity effect on STM (Meulman et al., 2012). In addition, it is possible that species-specific social and ecological factors - which we found unrelated to variation in task performance in captivity - would have a stronger effect in wild primate STM. A transfer to non-captive settings, however, would come with significant methodological, logistical and ethical challenges that would require collaboration beyond the ManyPrimates project.

Finally, the alignment between social and ecological predictor variables and phylogeny in our data limited the explanatory power of the modeling challenge. This alignment might have resulted partly from our opportunistic sampling strategy. Phylogenetic targeting (Arnold \& Nunn, 2010; MacLean et al., 2012) of species before data collection in future studies will help to overcome this limitation, as this approach allows identification of species that would increase the power to detect correlations between the task performance and certain species-level characteristics.

One of the aims of the present study, and of the ManyPrimates project more generally, is to standardize testing methodologies across species and institutions to obtain comparable data from large and diverse samples. This approach allows us to assess how comparable results for a given species are at different sites. Figure S5 in the supplementary material shows the performance of six species for which we collected sufficient data at at least 2 different sites. This comparison 
suggests that, even though there is variation across sites, species tend to perform on similar levels at different sites.

Despite our efforts to standardize data collection, there were some aspects of the testing protocol that could not be standardized across testing institutions. For example, subjects at some institutions were used to being separated from conspecifics for short periods of time and could be tested individually, whereas others had to be tested in their group or with their dependent offspring (see Supplementary Material). Furthermore, the group sizes of the tested individuals varied between and within species and across sites. Similarly, cup distance and board size could not be standardized across institutions due to the specific materials available at each site. However, we controlled for these variables in our analyses, and we did not find that substantive conclusions depend on the inclusion of these covariates. Another possible factor that could have influenced the subjects' task performance is the varying familiarity of the test subjects with the experimenters. However, given that this project was an intergroup-intersite collaboration, it was unavoidable and necessary that different experimenters collected the data at different locations.

The positive effect of task experience (i.e. previous participation in object choice tasks) on STM performance contrasts with the results of our pilot study, in which familiarity with object-choice tasks did not lead to a higher probability of success (ManyPrimates et al., 2019a). One potential explanation for such divergent results could be the more variable nature of the present dataset, both in terms of task experience and performance. Task experience can confer processing advantages by minimizing resource allocation to procedural details and thus maximizing resource allocation to relevant stimulus features. Recently, researchers have suggested that task experience may 
introduce a considerable bias in cross-species comparisons and may lead to unwarranted conclusions about the cognitive limitations of certain species, and thus, about cognitive evolution (see, e.g. Leavens et al., 2019). The importance of controlling for task experience is further underscored by the recent adoption of the so-called STRANGE (acronym for Social background; Trappability and self-selection; Rearing history; Acclimation and habituation; Natural changes in responsiveness; Genetic make-up; and Experience) framework within the field of ethological research (Rutz \& Webster, 2021).

The positive linear relation between STM performance and relative age runs against our predictions and previous studies of STM in humans and primates. Previous studies report an inverted U-curve relationship between STM capacity and age, whereby STM capacity is highest in young adults and decreases again with old age (Brockmole \& Logie, 2013, Darusman et al., 2014). Such divergent results could be explained by different sampling strategies (i.e., opportunistic sampling in our study as opposed to targeted sampling in studies addressing age-related changes in STM). Since an agerelated decline in STM capacity is typically reported for advanced ages (Brockmole \& Logie, 2013), it is possible that our results reflect an underrepresentation of such older individuals in our sample. Whether or not this was the case is difficult to say because our longevity estimates (which we used to norm age) might be biased due to differences in life expectancies between wild and captive populations. For understudied primates, these estimates are also likely to be imprecise.

\section{Conclusion}

The present study used the largest and most diverse sample of primates ever included in an experimental study, a scientific achievement only made possible by the large-scale international 
collaboration among scientists and institutions that represents the ManyPrimates project. This dataset allowed us to conduct robust phylogenetic analyses on the evolution of primate short-term memory as well as to evaluate how well species-specific social and ecological factors predict performance in a delayed-response task. When aggregating the data across species, we found that primates - as a group - performed well above chance in the delayed response task. Task success was found to decrease as the delay between stimuli presentation and response increased and previous task experience led to a higher probability of success in the task. Phylogenetic relatedness was found to strongly predict the observed variation in task performance among species whereas none of the species-specific ecological or social variables considered substantially contributed in explaining such variation. We encourage future studies to build upon the dataset and analyses of the present study by investigating the effects that other species-level predictors as well as individual-level predictors (such as rearing history, previous experience in cognitive tasks or hierarchical rank) have on STM. We want to emphasize again that such comprehensive evolutionary analyses are not possible with smaller or less diverse datasets and therefore crucially depend on the large-scale collaborative approach taken in this project. We therefore hope that the work presented here can act as a model for future studies to investigate the evolution of other cognitive abilities in the primate lineage. 
Acknowledgments

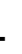

\section{Competing Interests}

The authors declare no competing interests. 


\section{References}

Altschul, D., Terrace, H., \& Weiss, A. (2016). Serial cognition and personality in macaques. Animal Behavior and Cognition, 3(1), 46-64. https://doi.org/10.12966/abc.02.04.2016

Arnold, C., Matthews, L. J., \& Nunn, C. L. (2010). The 10kTrees website: A new online resource for primate phylogeny. Evolutionary Anthropology: Issues, News, and Reviews, 19(3), 114-118. https://doi.org/10.1002/evan.20251

Arnold, C., \& Nunn, C. L. (2010). Phylogenetic targeting of research effort in Evolutionary Biology. American Naturalist, 176, 601-612. https://doi.org/10.1086/656490

Atkinson, R. C., \& Shiffrin, R. M. (1971). The control of short-term memory. Scientific American, 225(2), 82-90. https://doi.org/10.1038/scientificamerican0871-82

Barth, J., \& Call, J. (2006). Tracking the displacement of objects: A series of tasks with great apes (Pan troglodytes, Pan paniscus, Gorilla gorilla, and Pongo pygmaeus) and young children (Homo sapiens). Journal of Experimental Psychology: Animal Behavior Processes, 32(3), 239252. https://doi.org/10.1037/0097-7403.32.3.239

Bartus, R. T., Fleming, D., \& Johnson, H. R. (1978). Aging in the rhesus monkey: Debilitating effects on short-term memory. Journal of Gerontology, 33(6), 858-871. https://doi.org/10.1093/geronj/33.6.858 
Basile, B. M., \& Hampton, R. R. (2013). Dissociation of active working memory and passive recognition in rhesus monkeys. Cognition, 126(3), 391-396. https://doi.org/10.1016/j.cognition.2012.10.012

Basile, B. M., Schroeder, G. R., Brown, E. K., Templer, V. L., \& Hampton, R. R. (2015). Evaluation of seven hypotheses for metamemory performance in rhesus monkeys. Journal of Experimental Psychology. General, 144(1), 85-102. https://doi.org/10.1037/xge0000031

Beran, M. J., Perdue, B. M., Futch, S. E., Smith, J. D., Evans, T. A., \& Parrish, A. E. (2015). Go when you know: Chimpanzees' confidence movements reflect their responses in a computerized memory task. Cognition, 142, 236-246. https://doi.org/10.1016/j.cognition.2015.05.023

Blomberg, S. P., Garland, T. J., \& Ives, A. R. (2003). Testing for phylogenetic signal in comparative data: Behavioral traits are more labile. Evolution, 57, 717-745. https://doi.org/10.1111/j.0014-3820.2003.tb00285.

Boddy, A. M., McGowen, M. R., Sherwood, C. C., Grossman, L. I., Goodman, M., \& Wildman, D. E. (2012). Comparative analysis of encephalization in mammals reveals relaxed constraints on anthropoid primate and cetacean brain scaling. Journal of Evolutionary Biology, 25(5), 981-994. https://doi.org/10.1111/j.1420-9101.2012.02491.x

Brockmole, J. R., \& Logie, R. H. (2013). Age-related change in visual working memory: A study of 55,753 participants aged 8-75. Frontiers in Psychology, 4, 12. https://doi.org/10.3389/fpsyg.2013.00012 
Bunge, S. A. (2004). How we use rules to select actions: A review of evidence from cognitive neuroscience. Cognitive, Affective, \& Behavioral Neuroscience, 4(4), 564-579. https://doi.org/10.3758/cabn.4.4.564

Burkart, J. M., Hrdy, S. B., \& Van Schaik, C. P. (2009). Cooperative breeding and human cognitive evolution. Evolutionary Anthropology: Issues, News, and Reviews, 18(5), 175-186. https://doi.org/https://doi.org/10.1002/evan.20222

Bürkner, P. (2017). brms: An R package for Bayesian multilevel models using Stan. Journal of Statistical Software, 80(1), 1-28. https://doi.org/10.18637/jss.v080.i01

Burgoyne, A. P., \& Engle, R. W. (2020). Attention control: a cornerstone of higher-order cognition. Current Directions in Psychological Science, 29(6), 624-630. https://doi.org/10.1177/0963721420969371

Byrne, R. W. (1997). The Technical Intelligence hypothesis: An additional evolutionary stimulus to intelligence? In A. White \& R. W. Byrne (Eds.), Machiavellian Intelligence II: Extensions and Evaluations (pp. 289-311). Cambridge: Cambridge University Press.

Byrne, R. W., \& Whiten, A. (Eds.). (1988). Machiavellian intelligence: Social expertise and the evolution of intellect in monkeys, apes, and humans. Clarendon Press/Oxford University Press.

Cacchione, T., \& Rakoczy, H. (2017). Comparative metaphysics: thinking about objects in space and time. In J. Call, J. M. Burghardt, I. M. Pepperberg, C. T. Snowdon, \& T. Zentall (Eds.), APA Handbook of Comparative Psychology: Perception, Learning, and Cognition (pp. 579-599). American Psychological Association. 
Constantinidis, C., \& Procyk, E. (2004). The primate working memory networks. Cognitive, Affective \& Behavioral Neuroscience, 4(4), 444-465. https://doi.org/10.3758/cabn.4.4.444

Conway, A. R. A., Kane, M. J., \& Engle, R. W. (2003). Working memory capacity and its relation to general intelligence. Trends in Cognitive Sciences, 7(12), 547-552. https://doi.org/10.1016/j.tics.2003.10.005

Cowan, N. (2008). What are the differences between long-term, short-term, and working memory? Progress in Brain Research, 169, 323-338. https://doi.org/10.1016/S00796123(07)00020-9

Crockford, C., Wittig, R. M., Mundry, R., \& Zuberbühler, K. (2012). Wild chimpanzees inform ignorant group members of danger. Current Biology, 22(2), 142-146. https://doi.org/https://doi.org/10.1016/j.cub.2011.11.053

Cunningham, E., \& Janson, C. (2007). A socioecological perspective on primate cognition, past and present. Animal Cognition, 10(3), 273-281. https://doi.org/10.1007/s10071-0070078-3

Darusman, H. S., Call, J., Sajuthi, D., Schapiro, S. J., Gjedde, A., Kalliokoski, O., \& Hau, J. (2014). Delayed response task performance as a function of age in cynomolgus monkeys (Macaca fascicularis). Primates, 55(2), 259-267. https://doi.org/10.1007/s10329-013$0397-8$ 
DeCasien, A. R., Williams, S. A., \& Higham, J. P. (2017). Primate brain size is predicted by diet but not by sociality. Nature Ecology \& Evolution, 1(5), 0112. https://doi.org/10.1038/s41559-017-0112

Diamond, A., \& Doar, B. (1989). The performance of human infants on a measure of frontal cortex function, the delayed response task. Developmental Psychobiology, 22(3), 271-294. https://doi.org/10.1002/dev.420220307

Dunbar, R. I. M. (1998). The social brain hypothesis. Evolutionary Anthropology: Issues, News, and $\quad$ Reviews, $\quad 6(5), \quad$ 178-190. https://doi.org/10.1002/(SICI)15206505(1998)6:5<178::AID-EVAN5>3.0.CO;2-8

Dunbar, R. I. M., \& Shultz, S. (2007). Evolution in the social brain. Science, 317(5843), 1344 1347. https://doi.org/10.1126/science. 1145463

Egelkamp, C. L., Jacobson, S. L., Cronin, K. A., Wagner, K. E., Ross, S. R., \& Hopper, L. M. (2019). A comparison of sequential learning errors made by apes and monkeys reveals individual but not species differences in learning. International Journal of Comparative Psychology, 32. https://escholarship.org/uc/item/024578f5

Elmore, L. C., \& Wright, A. A. (2015). Monkey visual short-term memory directly compared to humans. Journal of Experimental Psychology: Animal Learning and Cognition, 41(1), 32-38. https://doi.org/10.1037/xan0000050

Engle, R. W. (2018). Working memory and executive attention: a revisit. Perspectives on Psychological Science, 13(2), 190-193.https://doi.org/10.1177/1745691617720478 
Geurten, M., Vincent, E., Van der Linden, M., Coyette, F., \& Meulemans, T. (2016). Working memory assessment: Construct validity of the Brown-Peterson Test. Canadian Journal of Behavioural Science / Revue Canadienne des Sciences du Comportement, 48(4), 328336. https://doi.org/10.1037/cbs0000057

Ghazizadeh, A., Griggs, W., Leopold, D. A., \& Hikosaka, O. (2018). Temporal-prefrontal cortical network for discrimination of valuable objects in long-term memory. Proceedings of the National Academy of Sciences of the USA, 115(9), 2135-2144. https://doi.org/10.1073/pnas.1707695115

Grimault, S., Robitaille, N., Grova, C., Lina, J.-M., Dubarry, A.-S., \& Jolicoeur, P. (2009). Oscillatory activity in parietal and dorsolateral prefrontal cortex during retention in visual short-term memory: Additive effects of spatial attention and memory load. Human Brain Mapping, 30(10), 3378-3392. https://doi.org/10.1002/hbm.20759

Harlow, H. F., \& Bromer, J. A. (1939). Comparative behavior of Primates. VIII. The capacity of Platyrrhine monkeys to solve delayed reaction tests. Journal of Comparative Psychology, 28(2), 299-304. https://doi.org/10.1037/h0053770

Harlow, H. F., Uehling, H., \& Maslow, A. H. (1932). Comparative behavior of primates. I. Delayed reaction tests on primates from the lemur to the orang-outan. Journal of Comparative Psychology, 13(3), 313-343. https://doi.org/10.1037/h0073864

Herculano-Houzel, S., Collins, C. E., Wong, P., \& Kaas, J. H. (2007). Cellular scaling rules for primate brains. Proceedings of the National Academy of Sciences of the USA, 104(9), 3562-3567. https://doi.org/10.1073/PNAS.0611396104 
Hopper, L. M., van de Waal, E., \& Caldwell, C. A. (2018). Celebrating the continued importance of "Machiavellian Intelligence" 30 years on. Journal of Comparative Psychology, 132(4), 427-431. https://doi.org/10.1037/com0000157

Hunter, W. S. (1913). The delayed reaction in animals and children. Animal Behavior Monographs, $2,1,86$.

Inoue, S., \& Matsuzawa, T. (2009). Acquisition and memory of sequence order in young and adult chimpanzees (Pan troglodytes). Animal Cognition, 12, 59-69. https://doi.org/10.1007/s10071-009-0274-4

Jolly, A. (1966). Lemur social behaviour and primate intelligence. Science, 153(3735), 501-506. https://doi.org/10.1126/science.153.3735.501

Jonides, J., Lewis, R. L., Nee, D. E., Lustig, C. A., Berman, M. G., \& Moore, K. S. (2008). The mind and brain of short-term memory. Annual Review of Psychology, 59, 193-224. https://doi.org/10.1146/annurev.psych.59.103006.093615

Kappeler, P. (1999). Convergence and divergence in primate social systems. In J. Fleagle, C. Janson, \& K. Reed (Eds.), Primate Communities (pp. 158-170). Cambridge: Cambridge University Press. https://doi:10.1017/CBO9780511542381.010

Kawai, N., \& Matsuzawa, T. (2000). Numerical memory span in a chimpanzee. Nature, 403(6765), 39-40. https://doi.org/10.1038/47405

Krupenye, C., \& Call, J. (2019). Theory of mind in animals: Current and future directions. WIREs Cognitive Science, 10(6), e1503. https://doi.org/https://doi.org/10.1002/wcs.1503 
Leavens, D. A., Bard, K. A., \& Hopkins, W. D. (2019). The mismeasure of ape social cognition. Animal Cognition, 22(4), 487-504. https://doi.org/10.1007/s10071-017-1119-1

Lewis, A., Berntsen, D., \& Call, J. (2019). Long-term memory of past events in great apes. Current Directions in Psychological Science, 28(2), 117-123. https://doi.org/10.1177/0963721418812781

MacLean, E. L. (2016). Unraveling the evolution of uniquely human cognition. Proceedings of the National Academy of Sciences of the USA, 113(23), 6348-6354. https://doi.org/10.1073/PNAS.1521270113

MacLean, E. L., Matthews, L. J., Hare, B. A., Nunn, C. L., Anderson, R. C., Aureli, F., Brannon, E. M., Call, J., Drea, C. M., Emery, N. J., Haun, D. B. M., Herrmann, E., Jacobs, L. F., Platt, M. L., Rosati, A. G., Sandel, A. A., Schroepfer, K. K., Seed, A. M., Tan, J., ... Wobber, V. (2012). How does cognition evolve? Phylogenetic comparative psychology. Animal Cognition, 15(2), 223-238. https://doi.org/10.1007/s10071-011-0448-8

Mansouri, F. A., Freedman, D. J., \& Buckley, M. J. (2020). Emergence of abstract rules in the primate brain. Nature Reviews Neuroscience, 21(11), 595-610. https://doi.org/10.1038/s41583-020-0364-5

ManyPrimates, Altschul, D., Bohn, M., Canteloup, C., Ebel, S. J., Hernández-Aguilar, R. A., Joly, M., Keupp, S., Petkov, C. I., Llorente, M., Proctor, D., Motes Rodrigo, A., Sutherland, K., Szabelska, A., Taylor, D., Völter, C. J., \& Wiggenhauser, N. G. (in press). ManyPrimates: Collaboration and open science initiatives in primate research. In M. J. 
Beran \& B. Schwartz (Eds.), Primate Cognitive Studies. Cambridge University Press. https://doi.org/10.31219/osf.io/7c93a

ManyPrimates, Altschul, D. M., Beran, M. J., Bohn, M., Call, J., DeTroy, S., Duguid, S. J., Egelkamp, C. L., Fichtel, C., Fischer, J., Flessert, M., Hanus, D., Haun, D. B. M., Haux, L. M., Hernandez-Aguilar, R. A., Herrmann, E., Hopper, L. M., Joly, M., Kano, F., ... Watzek, J. (2019a). Establishing an infrastructure for collaboration in primate cognition research. PLoS One, 14(10), e0223675. https://doi.org/10.1371/journal.pone.0223675

ManyPrimates, Altschul, D. M., Beran, M. J., Bohn, M., Caspar, K. R., Fichtel, C., Försterling, M., Grebe, N. M., Hernandez-Aguilar, R. A., Kwok, S. C., Llorente, M., Motes-Rodrigo, A., Proctor, D., Sánchez-Amaro, A., Simpson, E. A., Szabelska, A., Taylor, D., van der Mescht, J., Völter, C. J., \& Watzek, J. (2019b). Collaborative open science as a way to reproducibility and new insights in primate cognition research. Japan Psychological Research, 62, 205-220. https://doi.org/10.31234/osf.io/8w7zd

ManyPrimates, Géraud S. Aguenounon, S. B., Beaud, A., Bustamante, L., Canteloup, C., Joly, M., Loyant, L., Meunier, H., Roig, A., Troisi, C. A., \& Zablocki-Thomas, P. (2020). ManyPrimates: Une infrastructure de collaboration internationale dans la recherche en cognition des primates. Revue de Primatologie, 11. https://doi.org/10.4000/primatologie. 8808

Maslow, A. H., \& Harlow, H. F. (1932). Comparative behavior of primates. II. Delayed reaction tests on primates at Bronx Park Zoo. Journal of Comparative Psychology, 14(1), 97107. https://doi.org/10.1037/h0072093 
McElreath, R. (2018). Statistical rethinking: A Bayesian course with examples in $R$ and Stan. Chapman and Hall/CRC.

Mercer, T., \& Mckeown, D. (2014). Decay uncovered in nonverbal short-term memory. Psychonomic Bulletin \& Review, 21, 128-135. https://doi.org/10.3758/s13423-013$\underline{0472-6}$

Meulman, E. J., Sanz, C. M., Visalberghi, E., \& van Schaik, C. P. (2012). The role of terrestriality in promoting primate technology. Evolutionary Anthropology, 21(2), 58-68. https://doi.org/10.1002/evan.21304

Miller, G. A. (1956). The magical number seven, plus or minus two: some limits on our capacity for processing information. Psychological Review, 63(2), 81-97. https://doi.org/10.1037/h0043158

Milton, K. (1981). Distribution patterns of tropical plant foods as an evolutionary stimulus to primate mental development. American Anthropologist, 83(3), 534-548. https://doi.org/10.1525/aa.1981.83.3.02a00020

Mittermeier, R. A., Wilson, D. E., \& Rylands, A. B. (2013). Handbook of the mammals of the world. Volume 3: Primates. Lynx Edicions.

Morey, C. C., \& Bieler, M. (2013). Visual short-term memory always requires general attention. Psychonomic Bulletin \& Review, 20(1), 163-170. https://doi.org/10.3758/s13423-012$0313-\mathrm{z}$ 
Morey, R. D., \& Rouder, J. N. (2018). BayesFactor: Computation of Bayes Factors for Common Designs. $R \quad$ package version 0.9.12-4.2. https:/CRAN.Rproject.org/package $=$ BayesFactor.

Münkemüller, T., Lavergne, S., Bzeznik, B., Dray, S., Jombart, T., Schiffers, K., \& Thuiller, W. (2012). How to measure and test phylogenetic signal. Methods in Ecology and Evolution, 3(4), 743-756. https://doi.org/https://doi.org/10.1111/j.2041-210X.2012.00196.x

Miyake, A., Friedman, N. P., Emerson, M. J., Witzki, A. H., Howerter, A., \& Wager, T. D. (2000). The unity and diversity of executive functions and their contributions to complex "frontal lobe" tasks: a latent variable analysis. Cognitive Psychology, 41(1), 49-100. https://doi.org/10.1006/cogp.1999.0734

Nakahara, K., Hayashi, T., Konishi, S., \& Miyashita, Y. (2002). Functional MRI of macaque monkeys performing a cognitive set-shifting task. Science, 295(5559), 1532-1536. https://doi.org/10.1126/science.1067653

Nissen, H. W., Riesen, A. H., \& Nowlis, V. (1938). Delayed response and discrimination learning by chimpanzees. Journal of Comparative Psychology, 26(2), 361-386. https://doi.org/10.1037/h0056387

Osvath, M. (2009). Spontaneous planning for future stone throwing by a male chimpanzee. Current Biology, 19(5), 190-191. https://doi.org/10.1016/j.cub.2009.01.010

Oberauer, K. (2009). Design for a working memory. Psychology of Learning and Motivation, 51, 45-100. https://doi.org/10.1016/s0079-7421(09)51002-x 
Paradis, E., \& Schliep, K. (2019). Ape 5.0: An environment for modern phylogenetics and evolutionary analyses in R. Bioinformatics, 35(3), 526-528. https://doi.org/10.1093/bioinformatics/bty633

Petrides, M. (1995). Impairments on nonspatial self-ordered and externally ordered working memory tasks after lesions of the mid-dorsal part of the lateral frontal cortex in the monkey. The Journal of Neuroscience, 15(1), 359-375. https://doi.org/10.1523/jneurosci.15-01-00359.1995

Postle, B. R. (2015). The cognitive neuroscience of visual short-term memory. Current Opinion in Behavioral Sciences, 1, 40-46. https://doi.org/10.1016/j.cobeha.2014.08.004

Powell, L. E., Isler, K., \& Barton, R. A. (2017). Re-evaluating the link between brain size and behavioural ecology in primates. Proceedings of the Royal Society B: Biological Sciences, 284(1865), 20171765. https://doi.org/10.1098/rspb.2017.1765

Prétôt, L., \& Brosnan, S. F. (2019). Capuchin monkeys (Cebus [Sapajus] apella) show planning in a manual maze task. Journal of Comparative Psychology, 133(1), 81-91. https://doi.org/10.1037/com0000133

R Core Team. (2020). A language and environment for statistical computing. Vienna, Austria: R Foundation for Statistical Computing. https://www.r-project.org/.

Read, D. W. (2008). Working memory: A cognitive limit to non-human primate recursive thinking prior to Hominid evolution. Evolutionary Psychology, 6(4), 676-71. https://doi.org/10.1177/147470490800600413 
Resende, B., Ballesteros-Ardilla, A., Fragaszy, D., Visalberghi, E., \& Izar, P. (2021). Revisiting the fourth dimension of tool use: How objects become tools for capuchin monkeys. Evolutionary Human Sciences, 3, 18. https://doi.org/10.1017/ehs.2021.16

Riley, M. R., \& Constantinidis, C. (2016). Role of prefrontal persistent activity in working

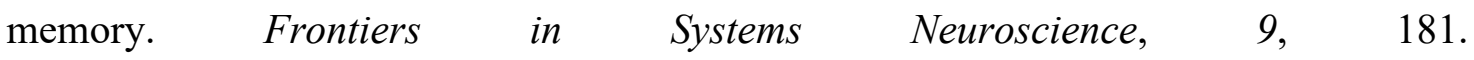
https://doi.org/10.3389/fnsys.2015.00181

Rosati, A. G. (2017). Foraging cognition: reviving the ecological intelligence hypothesis. Trends in Cognitive Sciences, 21(9), 691-702. https://doi.org/10.1016/j.tics.2017.05.011

Rosati, A. G., \& Santos, L. R. (2016). Spontaneous metacognition in rhesus monkeys. Psychological Science, 27(9), 1181-1191. https://doi.org/10.1177/0956797616653737

Rutz, C., \& Webster, M. M. (2021). Ethology adopts the STRANGE framework for animal behaviour research, to improve reporting standards. Ethology, 127(2), 99-101. https://doi.org/https://doi.org/10.1111/eth.13118

Sands, S. F., \& Wright, A. A. (1980). Primate memory: retention of serial list items by a rhesus monkey. Science, 209(4459), 938-940. https://doi.org/10.1126/science.6773143

Sanz, C. M., Call, J., \& Boesch, C. (Eds.). (2013). Tool use in animals: Cognition and ecology. Cambridge University Press.

Schwartz, B. L. (2019). Using natural ecology to predict higher cognition in human and non-human primates. Animal Behavior and Cognition, 6(4), 344-354. https://doi.org/10.26451/abc.06.04.13.2019 
Shettleworth, S. J. (2010). Cognition, evolution and behavior. Oxford University Press.

Shumaker, R. W., Walkup, K. R., \& Beck, B. B. (2011). Animal tool behavior. Johns Hopkins University Press.

Smuts, B. B., Cheney, D. L., Seyfarth, R. M., \& Wrangham, R. W. (Eds.). (1986). Primate societies. The University of Chicago Press.

Sol, D. (2009). Revisiting the cognitive buffer hypothesis for the evolution of large brains. Biology Letters, 5(1), 130-133. https://doi.org/10.1098/rsbl.2008.0621

Tomasello, M., \& Call, J. (1997). Primate cognition. Oxford University Press.

Unsworth, N., \& Engle, R. W. (2007). The nature of individual differences in working memory capacity: Active maintenance in primary memory and controlled search from secondary memory. Psychological Review, 114(1), 104-132. https://doi.org/10.1037/0033295x.114.1.104

Vallar, G., \& Papagno, C. (2002). Neuropsychological impairments of verbal short-term memory. In The Handbook of Memory Disorders (pp. 249-270). Wiley.

Völter, C. J., \& Call, J. (2017). Causal and inferential reasoning in animals. In J. Call, G. M. Burghardt, I. M. Pepperberg, C. T. Snowdon, \& T. Zentall (Eds.), APA Handbook of Comparative Psychology: Perception, Learning, and Cognition. (pp. 643-671). American Psychological Association. https://doi.org/10.1037/0000012-029 
Wise, S. (2017). The evolution of the prefrontal cortex in early primates and anthropoids. In J. H. Kaas (Ed.), Evolution of Nervous Systems (pp. 387-422). Academic Press. https://doi.org/10.1016/B978-0-12-820584-6.00028-3

Wynn, T., Hernandez-Aguilar, R. A., Marchant, L. F., \& Mcgrew, W. C. (2011). “An ape’s view of the Oldowan" revisited. Evolutionary Anthropology: Issues, News, and Reviews, 20(5), 181-197. https://doi.org/https://doi.org/10.1002/evan.20323

Zewald, J., \& Jacobs, I. (2021). Object permanence. In Encyclopedia of Animal Cognition and Behavior. Springer. https://doi.org/10.1007/978-3-319-47829-6_2103-1

Zola-Morgan, S., \& Squire, L. R. (1986). Memory impairment in monkeys following lesions limited to the hippocampus. Behavioral Neuroscience, 100(2), 155-160. https://doi.org/10.1037//0735-7044.100.2.155 


\section{Author contributions}

Conceptualization: Manuel Bohn (MB),

Investigation: Géraud Aguenounon (GA), Matthias Allritz (MA), Sébastien Ballesta (SB), Alice Beaud (AB), Manuel Bohn (MB), Sally L. Bornbusch (SLB), Angela Brandão (AB), Thomas Bugnyar (TB), Léa Bustamante (LB), Josep Call (JC), Chuangshi Cao (CC), Kai R. Caspar (KRC), Sarah E. DeTroy (SED), Shona Duguid (SD), Timothy M. Eppley (TME), Claudia Fichtel (CF), Juan Felipe Reátiga Parrish (JFRP), Chi Gong (CG), Nicholas M. Grebe (NMG), R. Adriana Hernandez-Aguilar (RAHA), Daniel Hanus (DH), Daniel B.M. Haun (DBMH), Lou M. Haux (LMH), Annabella Helman (AH), Istvan Hernadi (IH), Esther Herrmann (EH), Lydia M Hopper (LMH), Lei Huang (LH), Sarah M. Huskisson (SMH), Ivo Jacobs (IJ), Zhiyong Jin (ZJ), Marine Joly (MJ), Fumihiro Kano (FK), Stefanie Keupp (SK), Evelin Kiefer (EK), Balazs Knakker (BK), Katalin Koczan (KK), Larissa Kraus (LK), Sze Chai Kwok (SCK), Marie Lefrançois (ML), Elizabeth V. Lonsdorf (EVL), Louise Loyant (LL), Siyi Liu (SL), Katarzyna Majecka (KM), Hélène Meunier (HM), Luca Morino (LM), Flávia Mobili (FM), Alba MotesRodrigo (AMR), Caroline Nkoy Ihomi (CNI), Dariusz Pietraszewski (DP), Anthony Roig (AR), Vedrana Šlipogor (VŠ), Alejandro Sánchez-Amaro (ASA), Yutaro Sato (YS), Allie E. Schrock (AES), Amanda Seed (AMS), Caroline L. Shearer (CLS), Diana da Silva (DS), Yanjie Su (YS), Jingzhi Tan (JT), Christoph J. Völter (CJV), Elizabeth Warren (EW)

Data curation: Manuel Bohn (MB), Kai R. Caspar (KRC), Julia Watzek (JW), Yseult HéjjaBrichard (YHB), Alba Motes-Rodrigo (AMR), Vincent Nijman (VN), Camille A. Troisi (CAT), Luke Maurits (LM), Drew Altschul (DMA), Kirsten Sutherland (KS)

Methodology: Manuel Bohn (MB), Christoph J. Völter (CJV)

Formal analysis: Manuel Bohn (MB), Luke Maurits (LM)

Project administration: Manuel Bohn (MB), Christoph J. Völter (CJV), Julia Watzek (JW), Drew Altschul (DMA) 
Resources:

Visualization: Manuel Bohn (MB), James A. Grange (JAG), Julia Watzek (JW), Luke Maurits $(\mathrm{LM})$

Writing - original draft: Manuel Bohn (MB), Kai R. Caspar (KRC), Shona Duguid (SD), R. Adriana Hernandez-Aguilar (RAHA), Marine Joly (MJ), Miquel Llorente (ML), Alba MotesRodrigo (AMR), Tomas Persson (TP), Alejandro Sánchez-Amaro (ASA), Gabriela-Alina Sauciuc (GS), Manon K. Schweinfurth (MKS), Derry Taylor (DT), Christoph J. Völter (CJV), Pauline Zablocki-Thomas (PZT)

Writing - review \& editing: Manuel Bohn (MB), James Brooks (JB), Charlotte Canteloup (CC), Kai R. Caspar (KRC), Shona Duguid (SD), Timothy M. Eppley (TME), Claudia Fichtel (CF), Julia Fischer (JF), James A. Grange (JAG), Nicholas M. Grebe (NMG), Daniel B.M. Haun (DBMH), R. Adriana Hernandez-Aguilar (RAHA), Lydia M. Hopper (LMH), Ivo Jacobs (IJ), Marine Joly (MJ), Sze Chai Kwok (SCK), Miquel Llorente (ML), Julia Watzek (JW), Elizabeth V. Lonsdorf (EVL), Tomas Persson (TP), Alba Motes-Rodrigo (AMR), Manon K. Schweinfurth (MKS), Vedrana Šlipogor (VŠ), Alejandro Sánchez-Amaro (ASA), Gabriela-Alina Sauciuc (GS), Camille A. Troisi (CAT), Christoph J. Völter (CJV), Vincent Nijman (VN), Pauline ZablockiThomas (PZT), Laura Simone Lewis (LSL), Drew Altschul (DMA) 